\title{
In vivo photoprotection mechanisms observed from leaf spectral absorbance changes showing VIS-NIR slow-induced conformational pigment bed changes
}

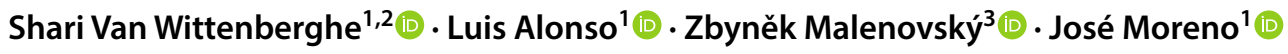

Received: 7 November 2018 / Accepted: 13 August 2019 / Published online: 20 September 2019

(c) The Author(s) 2019

\begin{abstract}
Regulated heat dissipation under excessive light comprises a complexity of mechanisms, whereby the supramolecular lightharvesting pigment-protein complex (LHC) shifts state from light harvesting towards heat dissipation, quenching the excess of photo-induced excitation energy in a non-photochemical way. Based on whole-leaf spectroscopy measuring upward and downward spectral radiance fluxes, we studied spectrally contiguous (hyperspectral) transient time series of absorbance $A(\lambda, t)$ and passively induced chlorophyll fluorescence $F(\lambda, t)$ dynamics of intact leaves in the visible and near-infrared wavelengths (VIS-NIR, 400-800 nm) after sudden strong natural-like illumination exposure. Besides light avoidance mechanism, we observed on absorbance signatures, calculated from simultaneous reflectance $R(\lambda, t)$ and transmittance $T(\lambda, t)$ measurements as $A(\lambda, t)=1-R(\lambda, t)-\mathrm{T}(\lambda, t)$, major dynamic events with specific onsets and kinetical behaviour. A consistent well-known fast carotenoid absorbance feature $(500-570 \mathrm{~nm})$ appears within the first seconds to minutes, seen from both the reflected (backscattered) and transmitted (forward scattered) radiance differences. Simultaneous fast Chl features are observed, either as an increased or decreased scattering behaviour during quick light adjustment consistent with re-organizations of the membrane. The carotenoid absorbance feature shows up simultaneously with a major $F$ decrease and corresponds to the xanthophyll conversion, as quick response to the proton gradient build-up. After xanthophyll conversion ( $t=3 \mathrm{~min}$ ), a kinetically slower but major and smooth absorbance increase was occasionally observed from the transmitted radiance measurements as wide peaks in the green $(\sim 550 \mathrm{~nm})$ and the near-infrared $(\sim 750 \mathrm{~nm})$ wavelengths, involving no further $F$ quenching. Surprisingly, in relation to the response to high light, this broad and consistent VIS-NIR feature indicates a slowly induced absorbance increase with a sigmoid kinetical behaviour. In analogy to sub-leaf-level observations, we suggest that this mechanism can be explained by a structure-induced low-energy-shifted energy redistribution involving both Car and Chl. These findings might pave the way towards a further non-invasive spectral investigation of antenna conformations and their relations with energy quenching at the intact leaf level, which is, in combination with $F$ measurements, of a high importance for assessing plant photosynthesis in vivo and in addition from remote observations.
\end{abstract}

Keywords Absorbance shift - Absorbed photosynthetic active radiation (APAR) - Controlled heat dissipation . Hyperspectral remote sensing $\cdot$ Non-photochemical quenching (NPQ) $\cdot$ Passive chlorophyll a fluorescence $\cdot$ Pigmentprotein dynamics

Electronic supplementary material The online version of this article (https://doi.org/10.1007/s11120-019-00664-3) contains supplementary material, which is available to authorized users.

Shari Van Wittenberghe

Shari.Wittenberghe@uv.es;

shari.vanwittenberghe@helsinki.fi

Extended author information available on the last page of the article

\section{Introduction}

Photosynthetic light-harvesting complexes (Lhcs) are sophisticated multichromophoric assemblies used to regulate and concentrate photo-excitations under wide-ranging incident irradiances for delivery to the reaction centres (Scholes et al. 2011). To protect themselves and the reaction centres from a potentially harmful solar irradiance excess, several regulated photoprotection mechanisms are activated at different time scales at the level of these complexes, balancing 
out the given energy supply. This regulated lowering of the excitation pressure on the reaction centres decreases photochemical quenching as energy is non-photochemically quenched inside the leaves by various physical and chemical signals (Demmig-Adams and Adams III 1992). The fast or so-called energy-dependent quenching (qE) mechanism has been assigned to thermal deactivation of singlet excited chlorophyll $\left(1 \mathrm{Ch} 1^{*}\right)$ in the antenna of photosystem II (PSII), lowering the quantum yield of fluorescence $(F)$ (Niyogi 1999). Although different mechanisms contribute to non-photochemical energy quenching (NPQ) in the shortterm, $\mathrm{qE}$ is typically presented as the dominating form of controlled energy dissipation in leaves under most natural conditions (Holzwarth et al. 2009). The qE-quenching mechanism reacts to the prevailing light conditions within few minutes. As the proton gradient $(\Delta \mathrm{pH})$ is built-up, it triggers chemical conversions as an immediate photoprotection response to light excess (Johnson and Ruban 2014). Additionally, a slower-induced photoprotection mechanism, often called the 'energy-independent' photoprotection takes place on a slower timescale but is suggested to have a higher photoprotection impact compared to the faster energy or $\Delta \mathrm{pH}$-dependent response (Nilkens et al. 2010; Jahns and Holzwarth 2012; Lambrev et al. 2012). Currently, there is still debate on how and where the $\Delta \mathrm{pH}$-dependent and $\Delta \mathrm{pH}$-independent mechanisms take place in the Lhcs associated with the two photosystems (PSI and PSII), forming the supramolecular light-harvesting pigment-protein complexes (PSI-LHCI and PSII-LHCII). Further, questions still remain on how these mechanisms are triggered, and how they occur in vivo for different plant species as they are typically indirectly observed both in vivo and in vitro through fluorescence dynamics and accompanying shifts.

Last 30 years of NPQ research proved the central role of carotenoids (Cars), the important accessory pigments of the Lhcs (Demmig et al. 1987; Demmig-Adams et al. 1995). Cars absorb light in the blue-to-green spectral range (with small shifts depending on the molecule) and subsequently transfer the energy by multiple interactions between the excited energy states of the different antenna pigments (Young and Frank 1996). The xanthophyll cycles were shown to be involved in both direct and indirect ways and in both the $\Delta \mathrm{pH}$-dependent and the $\Delta \mathrm{pH}-$ independent photoprotection mechanisms of the antenna, involving conformational changes in the Lhcs. Conformational changes are the structural changes within the Lhcs, which modulate the interaction between the chromophores (Ahn et al. 2008; Miloslavina et al. 2008; Müller et al. 2010; Jahns and Holzwarth 2012), i.e. the xanthophylls and chlorophyll, and the interaction between the chromophores and the protein scaffold itself (van Grondelle and Novoderezhkin 2006; Müh et al. 2010; Ostroumov et al. 2014). The well-known accumulation of zeaxanthin $(Z)$, as a result of lumen acidification initiating the enzymatic conversion of the Car violaxanthin (V) to zeaxanthin $(\mathrm{Z})$ via the intermediate antheraxanthin (A) in the VAZ xanthophyll cycle, is one of the short-term photoprotection mechanisms (seconds to minutes) (Bilger et al. 1989; Gamon et al. 1990; Peguero-Pina et al. 2013) that has been linked to several key processes triggering the NPQ mechanisms. After the chemical conversion into Z, which takes place in the lipid phase of the thylakoid membrane, the binding of $\mathrm{Z}$ to a Lhc induces a conformational change, which can be spectrally monitored by steady-state and time resolved $F$ methods (Moya et al. 2001; Dall'Osto et al. 2005). Hence, the most common conformational changes observed and discussed in literature are the $\mathrm{qE}$-dependent pigment-protein conformational changes that happen in the short term and drive the monomerization of the thylakoid membrane protein PS II subunit S PsbS (Deamer et al. 1967; Heber 1969; Bassi and Caffarri 2000; Moya et al. 2001; Ruban et al. 2007; Johnson et al. 2009a; Johnson and Ruban 2014; Krüger et al. 2014b). These PsbS-induced conformational changes involving aggregation and further a detachment of trimeric Lhc associated to PSII have been shown on a molecular basis (Correa-Galvis et al. 2016) and have been associated to low-energy (red-shifted) components in the absorption spectra of Lhcs (Johnson et al. 2009b). First zeaxanthin but later lutein was suggested to be involved in the further quick conformational changes, and held responsible for the triggering and maintaining of the structural protein folding (Croce et al. 1999; Bassi and Caffarri 2000). Despite these findings, the exact role of the different Cars in the antenna rearrangements remains controversial, mainly due to the lack of consensus on the molecular mechanisms in various conditions (Holt et al. 2004; Pascal et al. 2005; Jahns and Holzwarth 2012; Ruban et al. 2012). Overall, the Lhcs are shown to be effective probes of the quick conformational changes at the monomeric or trimeric unit (Johnson and Ruban 2009; Krüger et al. 2011, 2014a). However, little is known about the structural dynamics and transition between different conformational states available to the supramolecular PSII-LHCII and PSI-LHCI complexes being large ensembles of these Lhcs around their reaction centres.

Even slower induced organizational change of the Lhc, in which $Z$ is implied to have an allosteric role, has also been raised (Dall'Osto et al. 2005; Lambrev et al. 2012). Based on $F$ lifetime analysis it has been suggested that the Z-dependent conformational change takes place in the antenna proteins functionally connected to the PSII supercomplex and to the PSII reaction centre (Dall'Osto et al. 2005; Holzwarth et al. 2009), even though the molecular basis is unclear and in vivo observations are lacking. Holzwarth et al. (2009) suggested hereby that by binding to Lhc proteins into multiple sites, the accumulated $Z$ pool creates 
conditions for energy quenching by presumably modifying the hydrophobicity of the Lhc proteins, switching them into a further energy quenched state.

Several spectroscopy techniques, including transient absorption spectroscopy, fluorescence lifetime analysis, resonance Raman scattering, and circular dichroism, have observed and described in vitro significant reversible and quick reorganizations at the levels of (i) the ultrastructure of thylakoid membranes (Heber 1969; Krause 1973; Cseh et al. 2005), (ii) macro-organization of Lhcs within the membrane (Dall'Osto et al. 2005; Betterle et al. 2009), and (iii) purified Lhcs (Moya et al. 2001; Johnson and Ruban 2014). Although the relationship between NPQ and these different reorganizations remains to be further explored, provided evidence supports the idea that NPQ involves extensive reorganization of the Lhcs (Dall'Osto et al. 2005; Betterle et al. 2009; Garab 2016) opening energy dissipation channels that would trap the energy transferred amongst Chls (Krüger and van Grondelle 2017). However, as in vitro analysis of possible molecular mechanisms of NPQ are never performed on antenna complexes in their native environment (Krüger and van Grondelle 2017), the molecular basis and strengths of different energy-quenching mechanisms in vivo remains still unexplored (Holzwarth et al. 2009; Nilkens et al. 2010).

At the intact leaf level, absorbance changes related to NPQ have been often observed in parallel with additional scattering changes related to chloroplast movement (Bilger and Björkman 1990; Brugnoli and Björkman 1992; Kramer and Sacksteder 1998; Cazzaniga et al. 2013) or membrane rearrangements (Garab 2014), both complicating the disentanglement of the processes. These studies often focused on short-term (few minutes) changes observed commonly through a single wavelength, missing the dynamic behaviour of overlapping mechanisms. As an example, the spectral region of 500-570 $\mathrm{nm}$ has been exploited for the in vivo quantification of the VAZ xanthophyll cycle (Gamon et al. 1990; Evain et al. 2004), even though certain limitations have been noticed due to additional pigment bed changes (Ripullone et al. 2011). Hence, contiguous spectral data collections during sudden light transitions may allow for a more detailed observation of distinct spectral patterns during the light-induced reorganization of the antenna or entire chloroplasts. These may include a chromophore conversion (Gamon et al. 1990) and scattering changes due to chloroplast movement (Brugnoli and Björkman 1992), but also absorbance shifts due to changes in the local environment of the Lhcs (Johnson et al. 2009a), each displaying a specific spectral pattern. Due to this complexity, the specific contiguous spectral signatures linked to the various pigment-pigment interactions (including chemical conversions) and pigment-protein interactions (molecular rearrangements) are still poorly understood from in vivo observations and under natural-like illumination conditions. Distinguishing specific spectral signatures associated to distinct photo-avoidance and photoprotection processes would significantly advance the insights on the multichromophoric antenna dynamics upon light excess. Although optical leaf spectroradiometry dedicated to reflectance, transmittance and chlorophyll $F$ measurements under natural solar or natural-like illumination is still underexplored, it has the potential to study such dynamics in vivo in SI energy units (Van Wittenberghe et al. 2015; Alonso et al. 2017; Aasen et al. 2019).

Therefore, the goal of this study is to explore how different photo-avoidance and photoprotection mechanisms can be detected and followed from contiguous spectral radiance measurements in the visible (VIS, 400-700 nm) and nearinfrared (NIR, 700-800 nm) range. By analysing controlled dark-to-high-intensity light transients, our aim is to distinguish different spectral features from the information of bidirectional diffusively scattered and fluoresced light changes during the NPQ induction phase, if observable. With this, we intend to investigate the complexity of light interception and absorbance strategies by the antenna complexes of higher plants and the potential link with photoprotection dynamics in vivo, as this may serve as a further step in understanding vegetation dynamics from a remote sensing perspective.

\section{Materials and methods}

\section{Leaf clip and dual spectroradiometer set-up}

Hyperspectral signatures of leaf absorbance dynamics upon a high-intensity light (HL) adaptation were studied by measuring both upward and downward leaf diffusively scattered (reflected, transmitted), and diffusively emitted (fluoresced) radiance changes by means of two fibre optics, each attached to a spectroradiometer. Since all light not absorbed must be found as reflected (diffusively backscattered) or transmitted (diffusively forward scattered) light, while some absorbed light at a given wavelength may be emitted at longer wavelengths as fluorescence, a change in the overall hemispherical scattered light relates to a change in absorption or in fluorescence emission. We proposed a standardised laboratory set-up, in which single leaves attached to branches are clipped inside the custom-designed FluoWat leaf clip (Alonso et al. 2007; Van Wittenberghe et al. 2013) (Fig. 1) and mounted on a monopod ensuring a standardized portion of illumination entering the leaf clip chamber at an angle of $45^{\circ}$. A high-voltage LED (High Cri LED $10 \mathrm{~W}$ 17 V 3050-5900 K, Yuji International Co., Ltd, China), providing a broadband radiation spectrum between 400 and $780 \mathrm{~nm}$ with a peak in the blue part (Fig. 2a), was positioned towards the illumination port opening of the clip. To avoid overheating, the LED was glued on a heat sink and a small fan was attached for air-forced cooling. In front of 


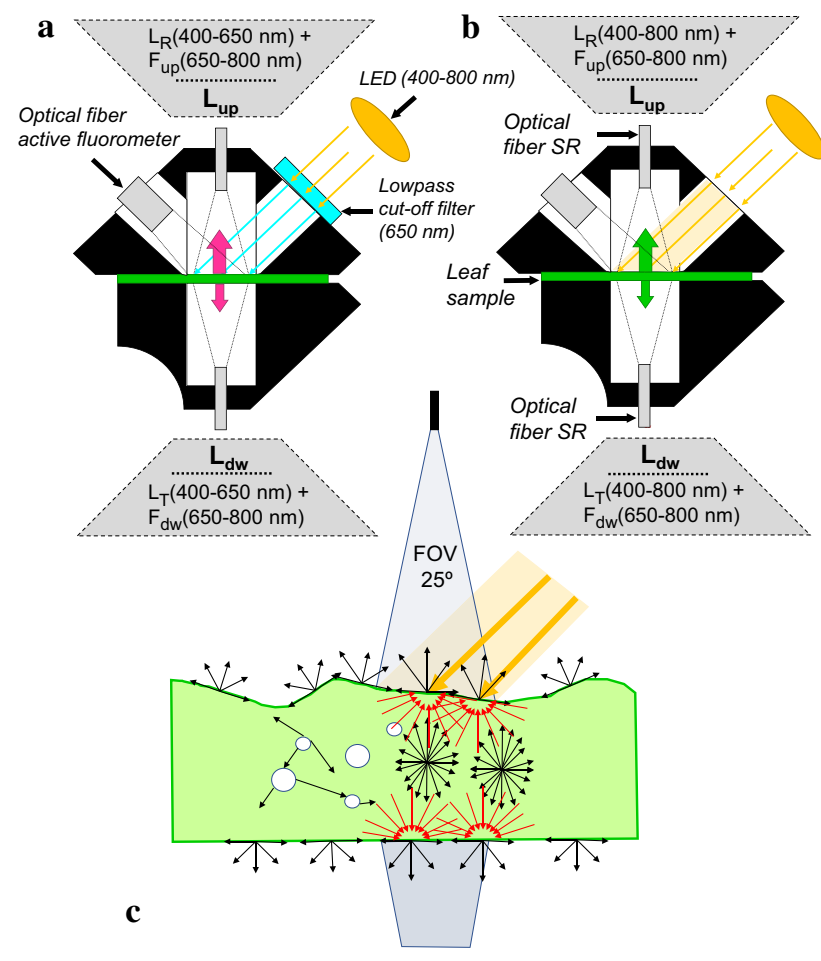

Fig. 1 Leaf clip set-up for spectral contiguous upward and downward radiance flux $\left(L_{\mathrm{up}}, L_{\mathrm{dw}}\right)$ measurements, with reflected and transmitted radiance $\left(L_{R}, L_{T}\right)$ and passive fluorescence emission $\left(F_{\mathrm{up}}, F_{\mathrm{dw}}\right)$ measurements under LED illumination using two spectroradiometers (SR), in combination with active $F$ measurements. The set-up with corresponding measured fluxes is given with (a, protocol 1) and without (b, protocol 2) the 650-nm low-pass filter, measuring the radiance fluxes in the diffusively scattered light coming from the leaf surface diffusively scattered light (red arrows, c)

the leaf-illumination opening, we attached a slider offering the three respective measuring options: (1) a dark cover, (2) a free opening, and (3) a high-performance OD4 lowpass filter (TechSpec, Edmund Optics GmbH, Germany) cutting off wavelengths $>650 \mathrm{~nm}$. The latter option allows measuring changes in pure $F$ emission between 650 and $800 \mathrm{~nm}$ (Figs. 1, 2b). The fibre optic of an ASD FieldSpec full-range (400-2500 nm) spectroradiometer (ASD Inc., Boulder, CO, USA) was inserted in the upper optical leaf clip opening (perpendicular direction to the leaf surface), while the second fibre optic of an ASD FieldSpec HandHeld 2 VNIR (325-1075 nm) spectroradiometer (ASD Inc., Boulder, CO, USA) was inserted in the bottom opening of leaf clip. This set-up allows simultaneous measurements of both upward and downward leaf radiance $\left(L_{\mathrm{up}}(\lambda), L_{\mathrm{dw}}(\lambda), \mathrm{W} \mathrm{m}^{-2}\right.$ $\mathrm{sr}^{-1} \mathrm{~nm}^{-1}$ ) measured respectively from the perpendicular directions to the adaxial and abaxial leaf side with a field of view (FOV) of $25^{\circ}$ (Fig. 1c), a spectral accuracy of $\pm 1 \mathrm{~nm}$ and a resolution of $<3 \mathrm{~nm}$. Leaves have both diffuse (nearLambertian) and specular (non-Lambertian) characteristics, defining the hemispherical scattered light (Myneni and
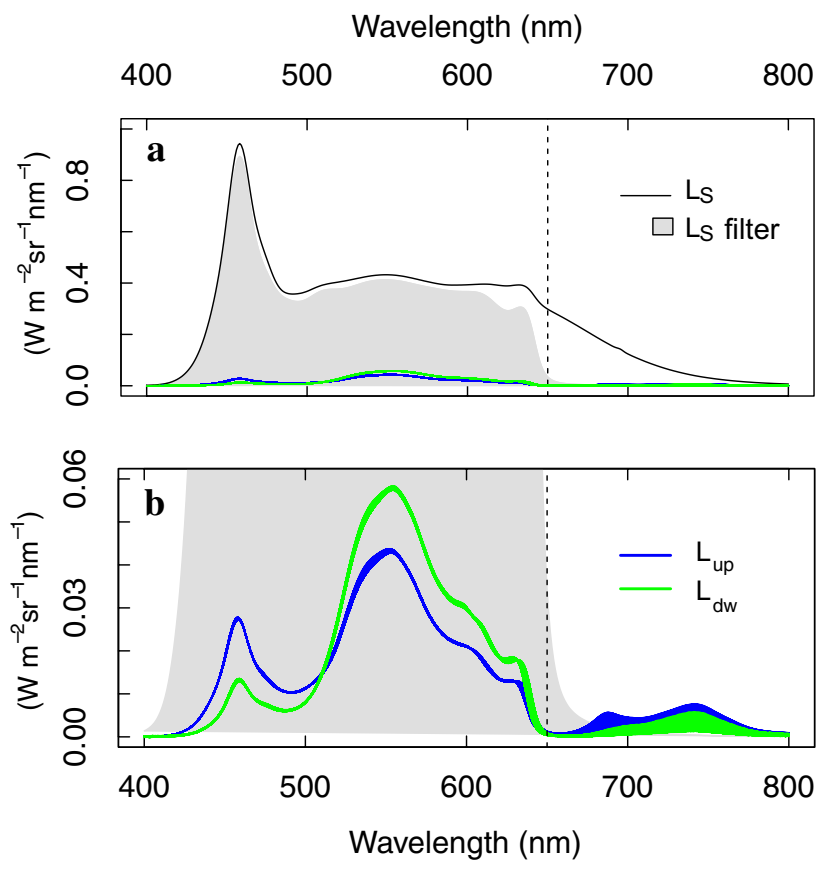

Fig. 2 Surface radiance $\left(L_{S}(\lambda)\right)$ without (black line) and with (grey area as integrated over the PAR region) the 650-nm cut-off filter (dashed line) constant in time (a), and the upward and downward leaf dynamic radiance $\left(L_{\mathrm{up}}(\lambda, t), L_{\mathrm{dw}}(\lambda, t)\right)$ measured during a darkto-high light intensity transient using a sudden light intensity of $1311 \mu \mathrm{mol} \mathrm{m}^{-2} \mathrm{~s}^{-1}\left(296 \mathrm{~W} \mathrm{~m}^{-2}\right)$ in the PAR region $(\mathbf{b})$

Ross 1991). Leaf transmittance, which only has the diffuse component, has a near-Lambertian distribution, while the distribution of reflected light depends on the incoming illumination angle. In our set-up, the $45^{\circ}$ illumination angle and nadir viewing with a $25^{\circ} \mathrm{FOV}$ angle is used to minimize the effects of angle dependency and to obtain a near-Lambertian approximation for the reflected light. Despite this, an offset of $3.7 \pm 1.0 \%$ in fAPAR, calculated for 30 leaves as the average of spectrally resolved absorbance in the region of photosynthetically active radiation (PAR, 400-700 nm) was found between our set-up and comparative integrating sphere measurements. This residual offset is mainly due to the lack of signal detection in the specular and neighbouring reflectance directions (i.e., specular component) of the incoming light by (1) the specific illumination and viewing geometry and (2) the selection of leaves with no extreme waxy or hairy surfaces. The diffuse character of the leaf is assumed to emanate primarily from the leaf interior, where each beam of light takes a unique path encountering different internal structures of varying geometric configurations and is scattered at each Refractive Index discontinuity (Kumar and Silva 1973). Hence, unlike measurements of homogenized in vitro suspensions (e.g. macromolecules in solution), where size of the particles might equal to the length of the measuring light wave and consequently produce an 
angularly anisotropic scattering, our in vivo optical measurements of leaves are angularly nearly isotropic, as the size of leaf-level scattering structures is larger than the measured wavelengths. In other words, the leaf surface behaves as a near-Lambertian scatterer, releasing prevailingly diffuse light originating from random multiple scattering within the ensemble of leaf interior elements. $L_{\text {up }}(\lambda)$ and $L_{\mathrm{dw}}(\lambda)$ are, respectively, composed of the reflected and transmitted radiance $\left(L_{R}(\lambda), L_{T}(\lambda), \mathrm{W} \mathrm{m}^{-2} \mathrm{sr}^{-1} \mathrm{~nm}^{-1}\right)$ in the $400-800$ nm range and the upward and downward $\mathrm{Chl}$ a $F$ emission $\left(F_{\text {up }}(\lambda)\right.$ and $\left.F_{\mathrm{dw}}(\lambda), \mathrm{W} \mathrm{m}^{-2} \mathrm{sr}^{-1} \mathrm{~nm}^{-1}\right)$ in the $650-800 \mathrm{~nm}$ region (Fig. 1a):

$L_{\text {up }}(400-800 \mathrm{~nm})=L_{R}(400-800 \mathrm{~nm})+F_{\text {up }}(650-800 \mathrm{~nm})$

$L_{\mathrm{dw}}(400-800 \mathrm{~nm})=L_{\mathrm{T}}(400-800 \mathrm{~nm})+F_{\mathrm{dw}}(650-800 \mathrm{~nm})$

The measuring fibre of an active fluorometer (PAM-2000, Walz GmbH, Effeltrich, Germany) can also be inserted in an opening opposite of the illumination opening (Fig. 1). Branches of Morus alba L. (White mulberry) and Juglans regia L. (Walnut) were freshly collected in June and July 2017 from the south-facing side of the trees, grown under a Mediterranean climate. Sun-grown leaves were chosen to select leaves optimized for an increased photoprotection capacity to excessive light. The branches were, immediately after cutting, submerged into water, transported to the lab, and kept in a dark place for $2 \mathrm{~h}$. Single-attached leaves were placed inside the leaf clip, while the dark cover slid was placed in front of the illumination opening. Both spectroradiometers were set to measure continuously single spectra with an integration time of 136 (FieldSpec) or 272 (HandHeld) ms. The reason for different integration times was a faster data collection of the HandHeld and our intention of collecting simultaneous $L_{\mathrm{up}}$ and $L_{\mathrm{dw}}$ spectra. Despite our efforts, the instruments' acquisitions could not be perfectly synchronized, resulting in need of proximate synchronization of both spectral transient series during data processing.

\section{Dark-to-high-intensity light-transient protocols}

A leaf under a saturating HL treatment typically converts V to $\mathrm{A}$ and to $\mathrm{Z}$ within approx. $10 \mathrm{~min}$ of high-intensity light (Bilger et al. 1989; Johnson et al. 2009a), although even shorter kinetical conversion steady-states have been measured at 3-5 min in vivo (Bilger et al. 1989; Peguero-Pina et al. 2013). In contrast, longer conversion steady-states around 60 min have been seen for different antenna subcomplexes in vitro (Heyde and Jahns 1998). A mathematical kinetic model description of VAZ conversion, originally applied for a system of liposome membranes (Latowski et al. 2000), has however, been functionally applied to the quick NPQ-transient phase, i.e. 0-2 min after dark to light exposure (D'Haese et al. 2004), kinetically indicating the quick VAZ conversion in vivo in the timescale of few minutes. Therefore, dark-to-HL transients of 10 min were applied to the leaves with an incoming LED illumination of approx. $270 \mathrm{~W} \mathrm{~m}^{-2}(1200 \mu \mathrm{mol}$ photons $\mathrm{m}^{-2} \mathrm{~s}^{-1}$ ) in the PAR region. Two different protocols were used to study the spectrally contiguous transient scattering and absorption dynamics. In the first protocol (protocol 1), the 650-nm cut-off low-pass filter was slid in front of the illumination opening, allowing to observe only $F$ emission in the 650-800 nm region (Fig. 1a). It is important to note that an internal photon scattering, taking place between the leaf and the filter, could potentially increase the radiance measured in the 620-650 region. This possible artefact is, however small, if being significant, due to the black and light-absorbing interior of the clip. Other possible artefacts due to the leaf clip design (e.g. filter transmission), the spectroradiometer (e.g. dark noise correction) and the set-up (e.g. stray light) were accounted for or diminished (Supplementary material S1). Any remaining instrumental artefact, even if small, can be considered constant in time and thus would not alter the variations in $L_{\text {up }}$ and $L_{\mathrm{dw}}$. Besides passive radiative measurements under LED illumination, additional active pulse-amplitude-mode (PAM) red-saturating flashes (400 ms, $7000 \mu$ mol photons $\mathrm{m}^{-2} \mathrm{~s}^{-1}$ ) were applied to derive the PAM-NPQ parameter based on broadband ( $710-850 \mathrm{~nm}$ ) relative $F$ changes as: $\left(\mathrm{Fm}-\mathrm{Fm}^{\prime}\right) /$ $\mathrm{Fm}^{\prime}$, where $\mathrm{Fm}^{\prime}$ is the maximum $F$ at each saturation pulse every $30 \mathrm{~s}$. After a dark adaptation of a minimum $2 \mathrm{~h}$, a saturation flash was given to determine Fo and Fm, respectively, the minimal and maximal fluorescence signal. One minute later, the dark-to-HL transient was executed, while $L_{\mathrm{up}}$ and $L_{\mathrm{dw}}$ spectra were continuously recorded (Fig. 2b).

During the second protocol (protocol 2), the 10-min dark-toHL transient was performed without low-pass filter in front of the illumination opening (Fig. 1b). Executing this protocol, $L_{R}$ and $L_{T}$ were measured over the VIS-NIR region $(400-800 \mathrm{~nm})$ and mixed with, respectively, $F_{\text {up }}$ and $F_{\mathrm{dw}}$ in the $650-800 \mathrm{~nm}$ region. Table 1 overviews the measured radiance fluxes and ratios calculated during the transient, implying a time dimension. After each transient protocol a Lambertian white spectral reference (Spectralon, Labsphere Inc., North Sutton, USA) was placed inside the leaf clip to measure the radiance arriving to the surface $\left(L_{\mathrm{S}}(\lambda, t), \mathrm{W} \mathrm{m}^{-2} \mathrm{sr}^{-1} \mathrm{~nm}^{-1}\right)$ in the LED spectral region which was tested to be stable through time (Fig. 2a). Changes in reflected and transmitted radiance $\left(\Delta L_{\mathrm{R}}(\lambda, \Delta t)\right.$ and $\Delta L_{\mathrm{T}}(\lambda, \Delta t)$, $\mathrm{W} \mathrm{m} \mathrm{m}^{-2} \mathrm{sr}^{-1} \mathrm{~nm}^{-1}$ ) are given a $\Delta t=t-t_{0}, t_{0}$ being the reference time chosen. Simultaneously $F$ emission changes $\left(\Delta F_{\text {up }}(\lambda, \Delta t)\right.$ and $\left.\Delta F_{\mathrm{dw}}(\lambda, \Delta t), \mathrm{W} \mathrm{m}^{-2} \mathrm{sr}^{-1} \mathrm{~nm}^{-1}\right)$ may occur. Hence, 
Table 1 Measured and calculated radiance fluxes and ratios with a wavelength $(\lambda)$ and time $(t)$ dimension for the leaf clip with dual spectroradiometer set-up

\begin{tabular}{|c|c|}
\hline Parameter & Definition \\
\hline$L(\lambda, t, \omega), \mathrm{W} \mathrm{m}^{-2} \mathrm{sr}^{-1} \mathrm{~nm}^{-1}$ & $\begin{array}{l}\text { Spectral radiance flux emitted, reflected, transmitted or received by a given surface, measured for a given angular } \\
\text { direction }\end{array}$ \\
\hline$L_{\text {up }}(\lambda, t), \mathrm{W} \mathrm{m}^{-2} \mathrm{sr}^{-1} \mathrm{~nm}^{-1}$ & Upward $L$, perpendicularly measured to the adaxial leaf surface or WR surface which is illuminated \\
\hline$L_{\mathrm{dw}}(\lambda, t), \mathrm{W} \mathrm{m} \mathrm{msr}^{-1} \mathrm{~nm}^{-1}$ & $\begin{array}{l}\text { Downward } L \text {, perpendicularly measured to the abaxial leaf surface or WR surface of which is illuminated from the } \\
\text { opposite site }\end{array}$ \\
\hline$L_{\mathrm{tot}}(\lambda, t), \mathrm{W} \mathrm{m}^{-2} \mathrm{sr}^{-1} \mathrm{~nm}^{-1}$ & Total $L, L_{\mathrm{up}}+L_{\mathrm{dw}}$ \\
\hline$L_{\mathrm{S}}(\lambda, t), \mathrm{W} \mathrm{m}^{-2} \mathrm{sr}^{-1} \mathrm{~nm}^{-1}$ & Radiance received by the surface, measured as $L_{\text {tot }}$ from the WR panel \\
\hline$L_{R}(\lambda, t), \mathrm{W} \mathrm{m}^{-2} \mathrm{sr}^{-1} \mathrm{~nm}^{-1}$ & $L_{\text {up }}$ component, diffusively scattered from the leaf surface (and its interior) without fluorescence \\
\hline$L_{\mathrm{T}}(\lambda, t), \mathrm{W} \mathrm{m} \mathrm{mr}^{-1} \mathrm{~nm}^{-1}$ & $L_{\mathrm{dw}}$ component, diffusively scattered from the leaf surface (and its interior) without fluorescence \\
\hline$R(\lambda, t),-$ & Reflectance, $L_{\mathrm{R}}(\lambda, t) / L_{S}(\lambda)$ \\
\hline$T(\lambda, t),-$ & Transmittance, $L_{T}(\lambda, t) / L_{S}(\lambda)$ \\
\hline$A(\lambda, t),-$ & Absorbance, $A(\lambda, t)=1-R(\lambda, t)-T(\lambda, t)$ \\
\hline$F_{\text {up }}(\lambda, t), \mathrm{W} \mathrm{m}^{-2} \mathrm{sr}^{-1} \mathrm{~nm}^{-1}$ & $L_{\mathrm{up}}$ component fluoresced from the leaf surface, given illumination to the adaxial side \\
\hline$F_{\mathrm{dw}}(\lambda, t), \mathrm{W} \mathrm{m}{ }^{-2} \mathrm{sr}^{-1} \mathrm{~nm}^{-1}$ & $L_{\mathrm{dw}}$ component fluoresced from the leaf surface, given illumination to the adaxial side \\
\hline$F_{\mathrm{tot}}(\lambda, t), \mathrm{W} \mathrm{m} \mathrm{sr}^{-2} \mathrm{nr}^{-1}$ & $\begin{array}{l}\text { Spectral radiant flux fluoresced from the abaxial and abaxial leaf surface, given illumination to the adaxial side, } F_{\text {up }} \\
+F_{\mathrm{dw}}\end{array}$ \\
\hline $\mathrm{FY}(t),-$ & Fluorescence yield, wavelength-integrated fluoresced flux (up, $\mathrm{dw}$ or tot) divided by $L_{S}$ in the PAR ( $400-700 \mathrm{~nm}$ ) \\
\hline
\end{tabular}

$$
\begin{aligned}
\Delta L_{\mathrm{up}}(400-800 \mathrm{~nm})= & \Delta L_{\mathrm{R}}(400-800 \mathrm{~nm}) \\
& +\Delta F_{\mathrm{up}}(650-800 \mathrm{~nm}) \\
\Delta L_{\mathrm{dw}}(400-800 \mathrm{~nm})= & \Delta L_{T}(400-800 \mathrm{~nm}) \\
& +\Delta F_{\mathrm{dw}}(650-800 \mathrm{~nm})
\end{aligned}
$$

And $\Delta \mathrm{L}_{\text {tot }}(400-800 \mathrm{~nm})=\Delta \mathrm{L}_{\text {up }}(400-800 \mathrm{~nm})$

$$
+\Delta \mathrm{L}_{\mathrm{dw}}(400-800 \mathrm{~nm})
$$

To investigate the leaf spectral dynamics during the induction phase of photoprotection, we calculated the spectral differences for different quenching transient phases by subtracting the first radiance spectrum from each consecutive transient radiance spectra of each phase. In the $400-650 \mathrm{~nm}$ spectral region where no $\mathrm{Chl} F$ emission nor $F$ emission change from any other component upon photoprotection dynamics occurs (Buschmann et al. 2000), reflectance and transmittance changes $(\Delta R$ and $\Delta T$ ) were calculated for $\Delta t=t-t_{0}$ by dividing $\Delta L_{\mathrm{R}}$ and $\Delta L_{\mathrm{T}}$ with corresponding $L_{\mathrm{S}}$ and used to derive the leaf absorbance change $(\Delta A)$ :

$$
\begin{aligned}
& \Delta R(\lambda, \Delta t)=\left(L_{R}(\lambda, t)-L_{R}\left(\lambda, t_{0}\right)\right) / L_{S}(\lambda) \\
& \Delta T(\lambda, \Delta t)=\left(L_{T}(\lambda, t)-L_{T}\left(\lambda, t_{0}\right)\right) / L_{S}(\lambda)
\end{aligned}
$$

Variations in surface feature irregularities with a size similar to the wavelength (leading to variable at-surface Mie scattering) are considered negligent compared to the variations in leaf-internal surfaces of varying geometric configurations inside the leaf. Hence, at-surface spectral changes are determined by the diffuse at-surface component rising from the random multiple scattering between the internal structures. This random and angularly independent character at leaf level is different from the possible variation in inpath Mie scattering during structural changes which may be considerable and detectable for single chloroplasts or other spherical leaf subsystems measured in solution due to significant horizontal light transport or strong light diffusion in the media (Latimer and Pyle 1972; Heirwegh et al. 1987). Nevertheless, although the at-leaf-surface diffuse component may be quasi-angularly anisotropic for each side, it is important to take into account the diffuse component of both adaxial and abaxial sides to account for the angular effects at leaf surface level determined by the overall internal scattering between geometrical arrangements and structures. Hence, our set-up results in an emanating approx. hemispherical leaf surface-leaving radiance insensitive to the in-path changes after multiple scattering (Fig. 1c), where total absorbance changes $(\Delta A)$ are only given by specific absorption changes. Consequently, in the absence of $F$, absorbance changes are estimated as the negative sum of changes in diffusively scattered illumination:

$\Delta A(\lambda)=-(\Delta R(\lambda)+\Delta T(\lambda))$,

whereby $\Delta A$ is given by the specific absorbance changes of the elements. Finally, the fluorescence yield (FY $(650-850 \mathrm{~nm})$, rel.) was calculated as the ratio of the spectrally integrated $F$ emission $(650-850 \mathrm{~nm})$ and the spectrally integrated absorbed PAR (400-700 nm) for, respectively, the upward, the downward and the total component $\left(\mathrm{FY}_{\text {up }}, \mathrm{FY}_{\mathrm{dw}}\right.$ and $\mathrm{FY}_{\text {tot }}$ ) (Van Wittenberghe et al. 2013). Spectral dynamics during the transients were investigated 
Fig. 3 Examples of quick (left column, a-d) and slow (right column, $\mathbf{e}-\mathbf{h}$ ) radiative changes $\left(\Delta L_{\mathrm{up}}, \Delta L_{\mathrm{dw}}, \Delta L_{\mathrm{tot}}, \mathrm{mW} \mathrm{m}^{-2}\right.$ $\mathrm{sr}^{-1} \mathrm{~nm}^{-1}$ ) from two different leaves measured using a $650-\mathrm{nm}$ cut-off filter (protocol 1), showing quick narrow overlapping $\Delta A$ features with dominant 500-570 nm feature (d) and slow broad decreasing $\Delta A$ features suggesting a possible slow chloroplast motion (grey area marks low irradiance range)
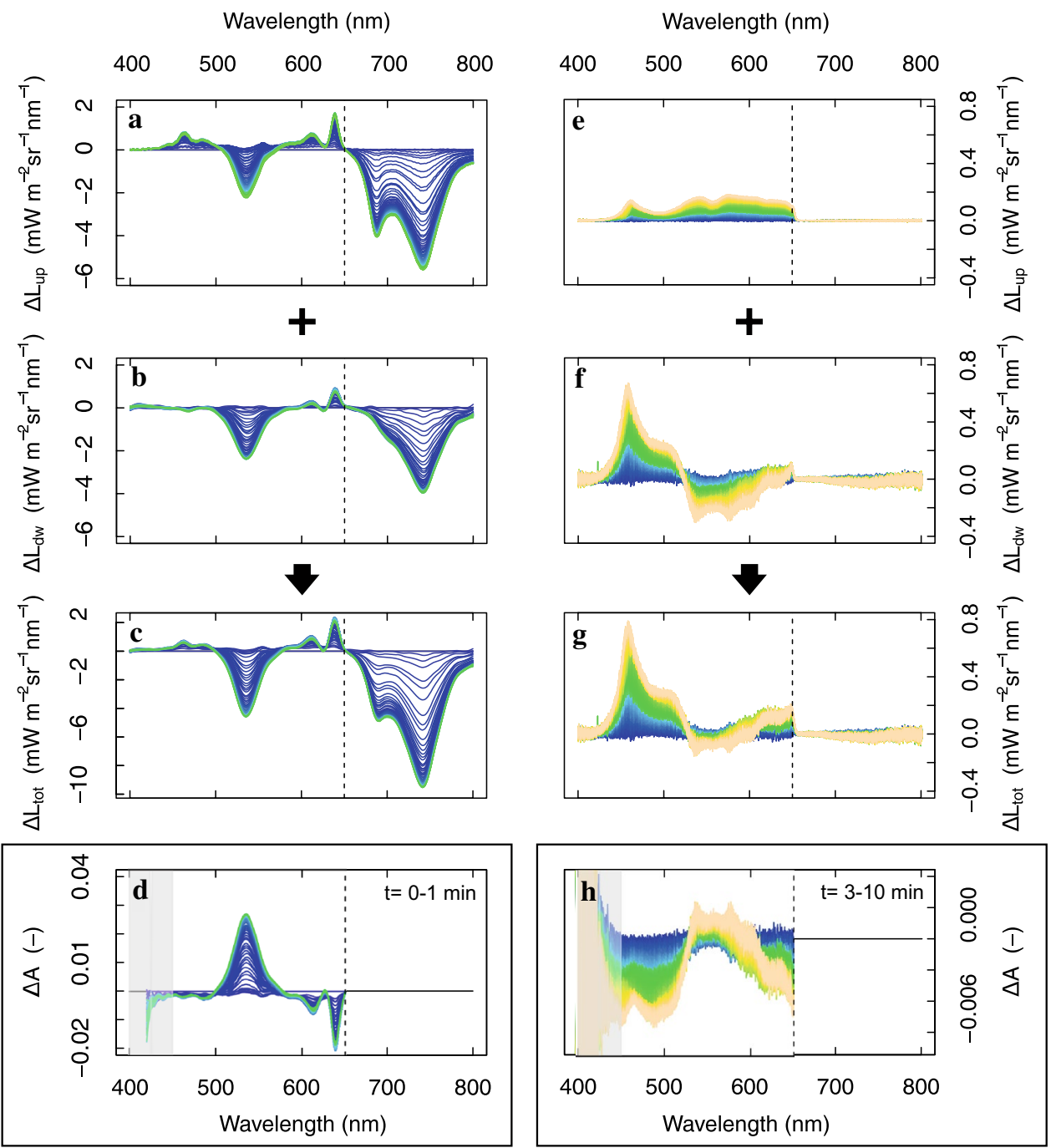

by analysing the maximal $\Delta R$ and $\Delta T$ and their shifts in time. Two spectral shifts of maximal $\Delta R$ and $\Delta T$ wavelength were used to analyse and to distinguish different absorption features during the light transients. All spectral processing and artwork were performed in the R software version 3.3.1 (www.r-project.org), making use of the asdreader library to import the spectral data. No spectral filters were used before data processing, only a local regression smoothing was occasionally applied for visual presentations.

\section{Results}

\section{Scattering versus absorbance increases}

A sudden interception of high-intensity illumination by the antenna causes the co-occurrence of several internal adjustment effects. Measured radiance changes are hereby wavelength-dependent and process-dependent, and may result from (1) a modified interception behaviour, e.g. by reorganizations at different hierarchical levels, i.e. at the level of thylakoid membrane or at the level of the entire chloroplast, and/or from (2) a modified absorbance behaviour, i.e. from molecular conversions or modified interactions between the chromophores. Both in the short ( $t=0-1 \mathrm{~min})$ and longer response ( $t=1-10 \mathrm{~min})$, several of these processes occur with distinct spectral responses (Fig. 3). Light avoidance is typically seen as an increased overall scattering to lower photon interception by the antenna. A case is shown within the short-term, fast narrow-featured scattering increases, in combination with scattering decreases (Fig. 3, left), which will be further discussed in the following section. In the longer response ( $t=1-10 \mathrm{~min})$, we occasionally observed stronger scattering increases, which were not necessarily conclusive from only forward or backscattered radiance changes, i.e. acting in the opposite direction in the green spectral region (Fig. 3b). This can be understood from the fact that scattering will depend on the overall change in orientation of the absorbing elements. When combining both upward and downward-scattered light, the total scattering 
increase from $\Delta L_{\text {tot }}$ shows the action spectra consistent with chloroplast motion (Brugnoli and Björkman 1992), being a rather slow process with a marked absorbance peak around $450 \mathrm{~nm}$ and shoulder around $475 \mathrm{~nm}$ (Inoue and Shibata 1973). A slight decrease in $F$ emission consistent with a lower chloroplast absorbance is simultaneously observed. In further results, we show examples in which slow chloroplast motion was only slightly or not observed at all, as it is not part of the regulated photoprotection and it complicates the detection of other features, which may be of interest.

\section{Fast $F$-coupled spectral dynamics during NPQ induction (protocol 1)}

Multiple fast narrow-featured spectral changes appear when applying the dark-to-HL protocol with the filter placed in front of the illumination opening, in parallel to immediate strong $F$ quenching as seen previously in Fig. 3 (left panel). Figure 4 shows the distinct spectral behaviour for separate $\Delta t$ phases during the transient of a single $M$. alba leaf, while Fig. 5 shows the overall behaviour of FY, PAM-NPQ, spectrally integrated $F_{\text {up }}$ versus spectrally integrated $L_{R}$ and for five leaves. Generally, a prompt decrease of upward and downward spectrally resolved fluorescence (Fig. 4a-d) and spectrally integrated FY is observed (Fig. 5a), followed by a much slower $F$ quenching until the end of the 10-min transient. Changes in $\mathrm{FY}_{\text {up }}$ are also reflected by the increase of the PAM-NPQ parameter, which saturates after approx. 4 min after sudden exposure to HL illumination (Fig. 5b). During the adaptation to HL, several changes in reflected and transmitted radiance were observed within the VIS (400-650 nm), some of them showing consistent decreases in scattering, while others showing opposite behaviour depending on the case (Figs. 3-left vs. 4). The largest $\Delta L_{R}$ and $\Delta L_{T}$ right upon light exposure were observed in the $500-570 \mathrm{~nm}$ region, showing consistent decreases for all observations. To illustrate the trend of this feature, the integral of the $\Delta L$ in this region was calculated as the difference of the radiance with the first spectrum $(t=0 \mathrm{~min})$, and normalized to the maximal difference, i.e., the integral of $\Delta L$ at $t=10 \mathrm{~min}$. For comparison with the co-occurring $F$ quenching, it was plotted against the co-occurring $F_{\text {up }}$ emission change in the 660-850 nm region (Fig. 5c). Several distinct response phases were distinguished based on the variable kinetic behaviours of both integrated $F_{\text {up }}(660-850 \mathrm{~nm})$ and $L_{\mathrm{R}}$ changes $(500-570 \mathrm{~nm})$ and presented in Fig. 4. Further, we observed a non-uniform $F$ quenching across the full spectral $F$ range. Figure 5d additionally demonstrates different phases of the red $(687 \mathrm{~nm})$ to far-red $(740 \mathrm{~nm}) F$ ratio evolution. A first small rapid rise of the F687/F740 ratio resulted in a local peak, which was followed by a steep decrease during the first $3 \mathrm{~min}$ and a mild, but steady, decrease in the following minutes.
Primary $F$ quenching involved only minor radiative changes in the VIS wavelengths (Fig. 4a-d). Nevertheless, the on-set of $F$ quenching took place rapidly in a period of seconds to 1 minute. An instant double-peak absorbance in the 500-570 $\mathrm{nm}$ region appeared quickly after illumination (Fig. 4a, b), followed by a $F$ quenching without any VIS spectral change (Figs. 4c, d, 5c). Subsequently, further leaf absorption changes in the 500-570 $\mathrm{nm}$ region re-occurred, seen from $\Delta L_{R}$ and $\Delta L_{T}$ decreases (Fig. $4 \mathrm{e}-\mathrm{h}$ ). Decrease in normalized radiance $(500-570 \mathrm{~nm})$ shows two differently steep quenching slopes (Fig. $5 \mathrm{c}$ ), i.e. initially relatively small $500-570 \mathrm{~nm}$ radiative changes with a strong $F$ quenching, followed by a stronger absorption in the $500-570 \mathrm{~nm}$ wavelengths accompanied by a strong $F$ quenching. Therefore, the two quenching phases were separated as presented in Fig. 4e-h. The initial radiative $500-570 \mathrm{~nm}$ changes, involving a high $F$ quenching, were characterized by a bell-shaped curve centred around $535 \mathrm{~nm}$ (Fig. 4e-f), while the following stronger changes extend slightly beyond $570 \mathrm{~nm}$ and accompanied by narrow-band features in the red region, with peaks located at 615 and $645 \mathrm{~nm}$, and minor features in the blue region (Fig. 4g, h). The red features became stronger during the next phase, while additional VIS features seem to overlap the 500-570 nm feature (Fig. 4i, j). Ultimately, spectral changes during the last phase $(t=200-600 \mathrm{~s})$ showed a negative-to-positive twist in 520-600 $\mathrm{nm}$ absorbance values and weakening of the red features, while the overall scattering in the VIS increased (Fig. 4k, 1).

Although some variable scattering behaviour could be detected in spectral regions where absorption of several foliar pigments overlaps (i.e. $400-500 \mathrm{~nm}$ ), the dynamic changes in the region where photoprotection-related spectral features occur (i.e. $500-570 \mathrm{~nm}$ ) were found to be time-consistent in the first 3 minutes upon illumination for five different leaves tested (Fig. 5c). This can be seen across different examined plant species tested here and in other works (Van Wittenberghe et al. 2018; Vilfan et al. 2018). Reflectance and transmittance changes of the shown feature occurred in equal magnitude pointing to a true absorbance increase. Other consecutive or simultaneous minor scattering changes within the full VIS spectrum (Fig. 4k, 1) were not every time strongly or consistently detected (Figs. 3-left vs. 4). Hence, additional fast heterogeneous spectral changes occurred. Taking the reflectance changes at $535 \mathrm{~nm}$ as reference wavelength, only the changes in the 500-570 nm region resulted highly correlated during $t=0-20 \mathrm{~s}$ (Fig. 6a), while changes the first minutes hereafter $(t=20-180 \mathrm{~s})$ were strongly correlated with the entire $500-570 \mathrm{~nm}$ region as well as with the 600-620 and 630-650 nm regions (Fig. 6b). After $3 \mathrm{~min}$, when the $500-570 \mathrm{~nm}$ feature has terminated its activity, various spectral changes in the $430-650 \mathrm{~nm}$ region occur, showing further complex behaviour during light adaptation (Fig. 6c). 

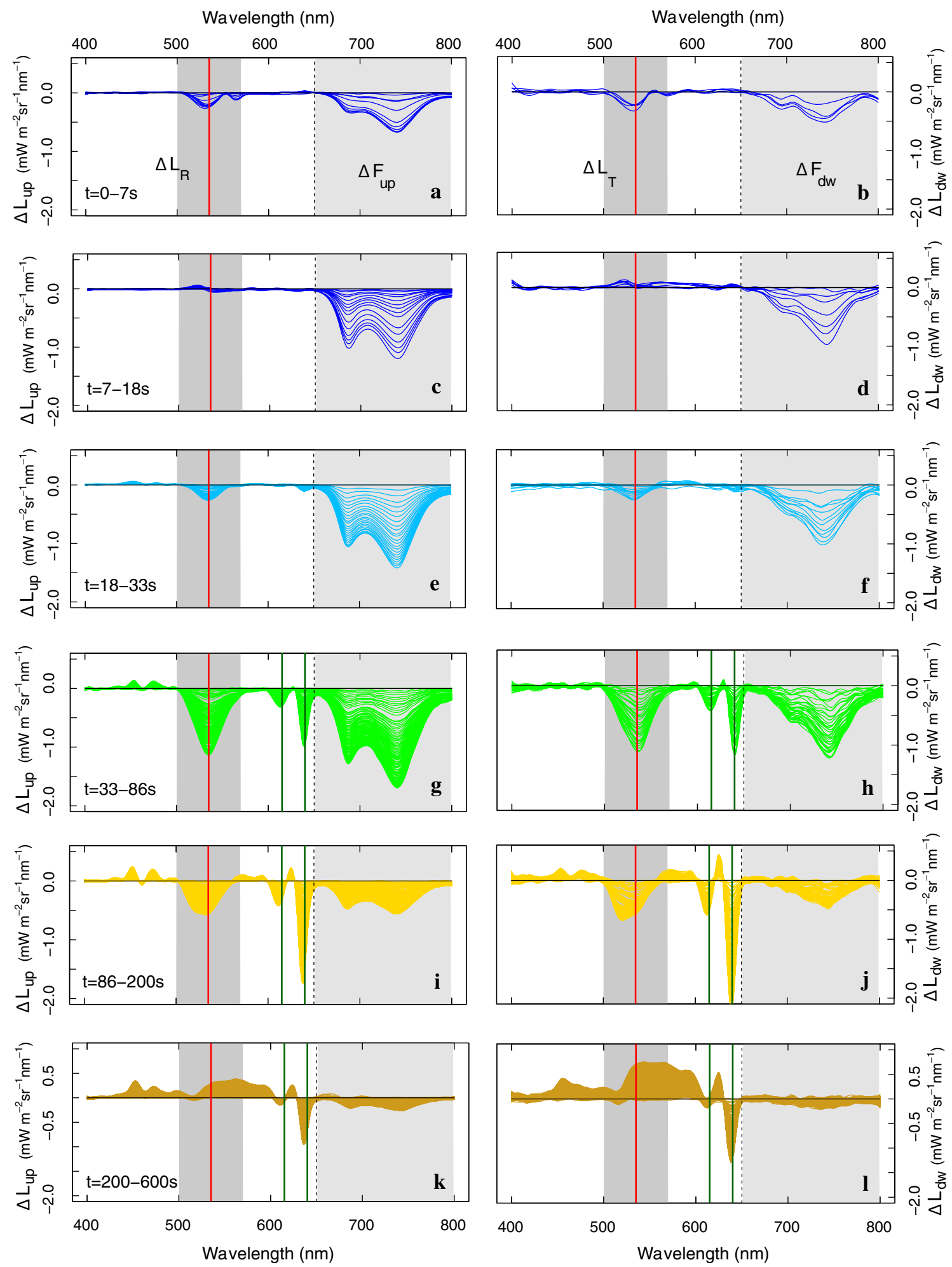

Fig. 4 Upward (left column) and downward (right column) radiative change $\left(\Delta L_{\text {up }}\right.$ and $\left.\Delta L_{\mathrm{dw}}, \mathrm{mW} \mathrm{m}^{-2} \mathrm{sr}^{-1} \mathrm{~nm}^{-1}\right)$, with reflected and transmitted changes $\left(\Delta L_{\mathrm{R}}\right.$ and $\left.\Delta L_{T}\right)$ in the VIS $(400-650 \mathrm{~nm})$ and $F$ emission changes in the red and far-red $(650-800 \mathrm{~nm}$, light grey shade) of a $M$. alba leaf for different photoprotection phases during the 10-min light transient $\left(1200 \mu \mathrm{mol} \mathrm{m}^{-2} \mathrm{~s}^{-1}\right)$, when using a 650 -

nm cut-off filter (dashed line). The reference spectrum is the first spectrum of each phase, respectively, showing only radiative changes for each specified period. The highly dynamic spectral region of $500-570 \mathrm{~nm}$ is marked in a dark grey shade. Major absorption wavelengths are marked as follows: red $(531 \mathrm{~nm})$ and dark green (615 and $645 \mathrm{~nm})$ 

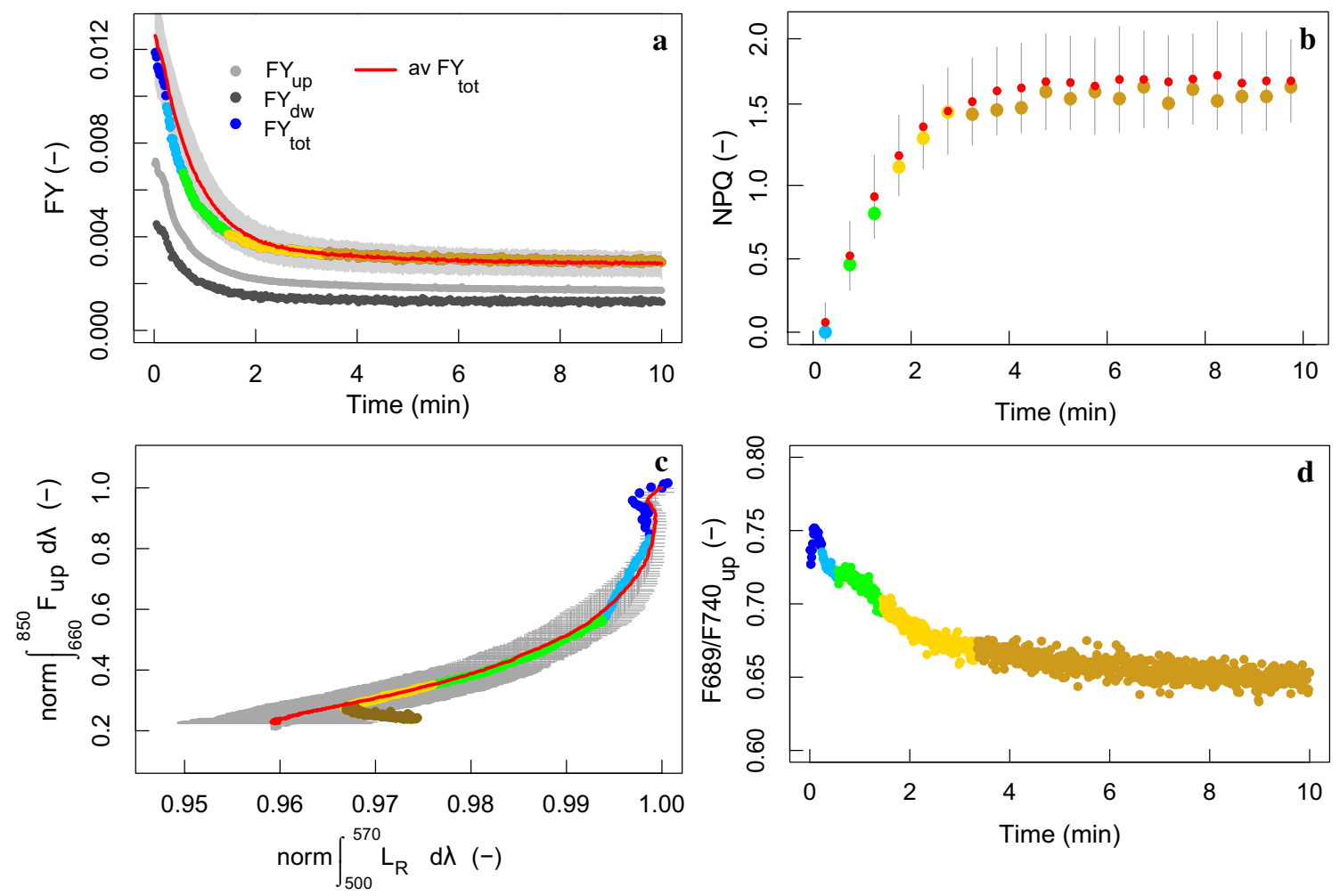

Fig. 5 Upward, downward and total fluorescence yield (FY, rel.). (a), non-photochemical fluorescence quenching (PAM-NPQ) (b), normalized difference of the integrated upward fluorescence $\left(F_{\text {up }}\right.$, $660-850 \mathrm{~nm})$ in respect to $t=0$ min versus normalized difference of the integrated reflected radiance $\left(L_{R}, 500-570 \mathrm{~nm}\right)$ in respect to $t=0 \mathrm{~min}(\mathbf{c})$, and upward fluorescence peak ratio $\mathrm{F} 689 / \mathrm{F} 740$ (d) presented for the 10-min dark-to-high-intensity light transient given

\section{Slow $F$-uncoupled spectral dynamics during NPQ induction (protocol 1)}

For both species, several leaves subjected to the dark-toHL transient showed more pronounced scattering changes, in comparison to the results presented in the sect. "Scattering versus absorbance increases". Figure 7 presents radiative evolution for a $M$. alba leaf upon a 10-min transient. It shows the upward and downward radiance spectra $(\mathrm{a}-\mathrm{b})$ and the respective changes $\left(\Delta L_{\mathrm{up}}\right.$ and $\left.\Delta L_{\mathrm{dw}}\right)$, using the spectrum at $t=0 \mathrm{~min}$ as the reference $(\mathrm{c}-\mathrm{d})$. Fast radiative changes in the green $(500-570 \mathrm{~nm})$ and red $(615 \mathrm{~nm})$ wavelengths, which were previously shown in Fig. 4, were observed from the upward and downward signal during the first 2 min after illumination (blue-drawn spectra). Simultaneously, $F$ was rapidly quenched. Further significant radiative changes were observed in $L_{T}$ from $t=5 \mathrm{~min}$ onward (lime-drawn spectra), following the prior fast changes (Fig. 7d). Compared to the previously observed green absorbance change, this consecutive and slower decrease in scattering, only seen from decrease of $\Delta T$, is stronger and, moreover, taking place to the M. alba leaf presented in Fig. 3. Colours indicate the different phases during the transient as presented in Fig. 3, which are distinguished based on the variable kinetic behaviour of both $F_{\text {up }}$ $(660-850 \mathrm{~nm})$ and $L_{R}$ changes $(500-570 \mathrm{~nm})$. Additional average (in red) and standard deviation (in grey) values are computed for five dark-adapted leaves measured under identical illumination conditions $\left(1200 \pm 65 \mu \mathrm{mol} \mathrm{m}{ }^{-2} \mathrm{~s}^{-1}\right)(\mathbf{a}-\mathbf{c})$

across the full VIS spectral range (until $650 \mathrm{~nm}$ where illumination was cut-off). Surprisingly, a strong total decrease in scattering is observed here, which intuitively contradicts with a light-avoidance strategy. i.e. as given by chloroplast motion (Fig. 3right). No further simultaneous $F$ quenching was measured during these changes after $t=2 \mathrm{~min}$. The evolution of the wavelength with a maximal change during the light adaptation in the 430-600 nm region is shown for $\Delta R$ (Fig. 7e) and for $\Delta T$ (Fig. 7f). The $\Delta R$ peak maximum shifts slightly from 537 to $533 \mathrm{~nm}$ during the first $2 \mathrm{~min}$ and it remains stable hereafter. $\Delta T$ showed a clear peak wavelength shift to longer wavelengths during $t=5-10 \mathrm{~min}$, i.e. from approx. 534 to $539 \mathrm{~nm}$, indicating an additional absorbance feature appearance with different maximum location.

To separate the $F$-coupled and $F$-uncoupled absorbance features observed from $\Delta R$ and $\Delta T$, a new reference spectrum was taken at $t=3 \mathrm{~min}$ for calculation of changes in both $R$ and $T$, i.e. before the maximum peak shifts shown in Fig. $7 \mathrm{~g}$, h, and consequently subtracted from the consecutive dynamic radiance signal. The fast or $\Delta \mathrm{pH}$ dependent absorbance changes $(t=0-3 \mathrm{~min})$ were almost 

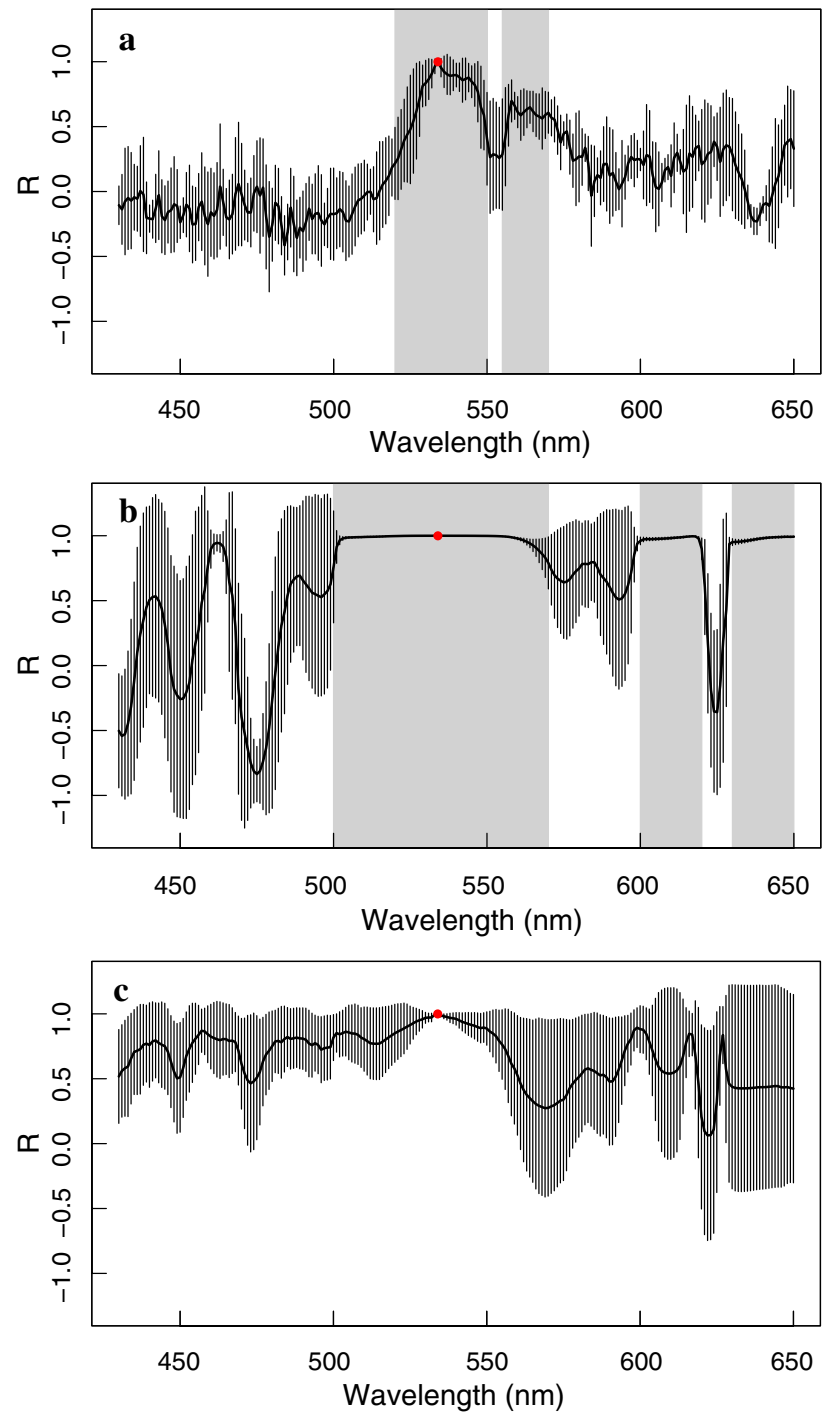

Fig. 6 Correlation coefficient $(R) \pm$ standard deviation between $\Delta R$ at $535 \mathrm{~nm}$ (red point) with the reflectance wavelength changes (430$650 \mathrm{~nm}$ ) during $t=0-20 \mathrm{~s} \mathrm{(a),} t=20-180 \mathrm{~s}(\mathbf{b})$, and $t=180-600 \mathrm{~s}$ (c) for five $M$. alba leaves exposed to a high-intensity illumination $\left(1162 \pm 30 \mu \mathrm{mol} \mathrm{m} \mathrm{m}^{-2} \mathrm{~s}^{-1}\right)$; stand-out regions of high correlation are indicated with grey areas in $\mathbf{a}, \mathbf{b}$

equally represented in shape and magnitude of $\Delta R$ and $\Delta T$ (Fig. 8a, c). A minor absorbance peak shift towards shorter wavelengths ( $\Delta R: 537$ to $532 \mathrm{~nm} ; \Delta T: 535$ to $533 \mathrm{~nm}$ ) indicates the existence of more than one A feature during these early fast changes. Slow absorbance changes $(t=3-10 \mathrm{~min})$ appeared to be minor in terms of the $\Delta R$, but clearly observable from the $\Delta T$ spectra (Fig. $8 \mathrm{~b}, \mathrm{~d}$ ). Figure $8 \mathrm{~d}$ shows an additional $\Delta T$ decrease of $2 \%$ during this period. The maximum $\Delta T$ decrease located at 554-555 $\mathrm{nm}$ remained stable, suggesting existence of a single absorbance feature at that wavelength during the given transient period. Due to the use of a cut-off filter, the full red and near-infrared spectral changes until $800 \mathrm{~nm}$ could not be shown. Calculation of the absorbance $(A)$ and the absorbance changes $(\Delta A)$ and their separation into fast and slow absorbance features for the $430-620 \mathrm{~nm}$ region measured during the 10-min transient period are shown in Fig. 9. The fast $\Delta A$ showed a pronounced absorption feature in the green wavelengths (maximum at $533 \mathrm{~nm}$, vertical dotted line), whereas the slow $\Delta A$ revealed a broadened absorbance peak shifted towards longer wavelengths, with a maximum at $553 \mathrm{~nm}$ (vertical red solid line). Both peaks showed an equal $2 \%$ increase in absorbance at their respective peak wavelength, showing a $\Delta \lambda=20 \mathrm{~nm}$. Moreover, it seems that the slow $\Delta A$ extend beyond $620 \mathrm{~nm}$.

\section{Fast and slowly induced absorbance features in the VIS and NIR (protocol 2)}

In accordance with the previous observations, we observed identical fast and slow absorbance changes for J. regia leaves with a consistent spectral action feature. Measuring the optical properties with (protocol 1) and without filter (protocol 2) allowed observing the spectral changes beyond $620 \mathrm{~nm}$, which are composed of both absorbance and $F$ changes (Fig. 10). When using the filter, the measurements reconfirmed that fast absorbance changes, in immediate response to the $\Delta \mathrm{pH}$, coincide with strong $F$ quenching, while slow absorbance changes show no further additional $F$ quenching (Fig. 10a), as demonstrated before (Fig. 7d). This effect is better seen by plotting the spectral integrals of the two spectral intervals, i.e. $500-570$ and $660-850 \mathrm{~nm}$ (Fig. 10c) where $F$ varies together with the $L_{\text {tot }}$ at the beginning ( $t=0-3 \mathrm{~min}$ ) of the transient (blue dots), but then it reaches stability, whereas $L_{\text {tot }}(500-570 \mathrm{~nm})$ keeps on evolving. Figure $10 \mathrm{~d}$ shows the same, a similar trend when the filter is not used, but now the integral in the NIR captures both contributions, i.e. emitted $F$ and scattered radiance; as a result, $L_{\text {tot }}$ in the NIR radiance keeps evolving after $t=3 \mathrm{~min}$. To assure that no significant quick scattering changes are detected beyond $650 \mathrm{~nm}$ (which cannot be observed when using the filter), it was tested that for both cases the F quenching was happening at the same relative speed. This test ensures that no further significant fast absorbance or scattering changes could be detected beyond $650 \mathrm{~nm}$, which cannot be observed when using the filter. The result showing the same relative $F$-quenching speed for the $500-570 \mathrm{~nm}$ integral in both cases (Fig. 10e) suggests that all fast spectral changes $>650 \mathrm{~nm}$ can be, in the unfiltered case, attributed to $F$ quenching only. On the contrary, the slow absorbance changes $(t=4-10 \mathrm{~min})$ did not co-occur with further $F$ quenching (Fig. 10c). All additional spectral changes beyond $650 \mathrm{~nm}$ show a perfect linear relationship with the VIS spectral changes, suggesting that they belong to the same process.

To verify this further, the spectral signatures of both processes with different onset, were further analysed in 


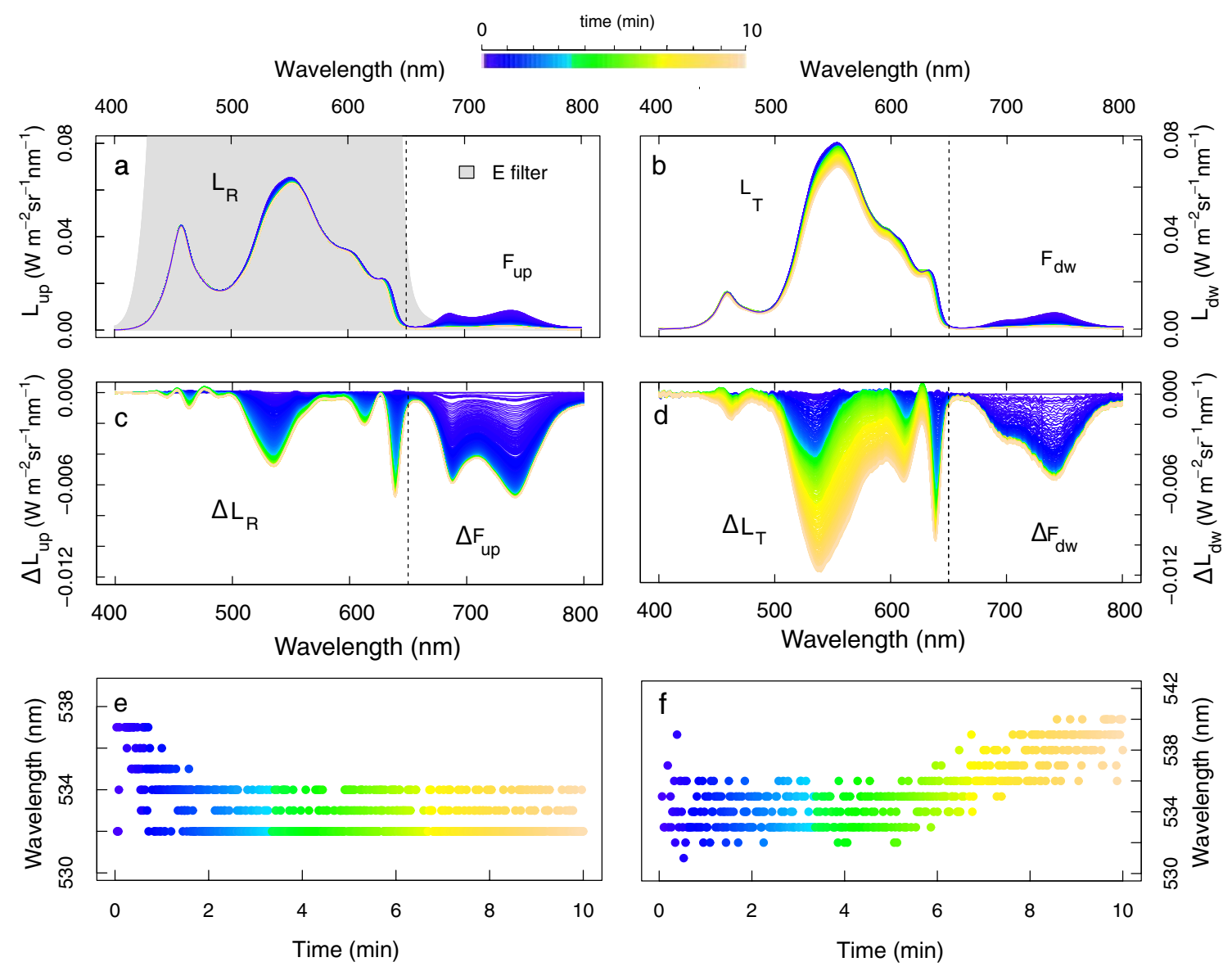

Fig. 7 Upward and downward radiance $\left(L_{\text {up }}\right.$ and $\left.L_{\mathrm{dw}}\right)$ and $F$ emission $\left(F_{\mathrm{up}}, F_{\mathrm{dw}}\right)$ of a dark-adapted M. alba leaf measured upon sudden high-intensity LED illumination $\left(1311 \mu \mathrm{mol} \mathrm{m} \mathrm{m}^{-2} \mathrm{~s}^{-1}\right.$ or $296 \mathrm{~W} \mathrm{~m}^{-2}$ in the PAR region, partly illustrated as grey shade) during a 10-min transient and the filter protocol $(\mathbf{a}, \mathbf{b})$. Transient changes are displayed

the spectral ranges of $500-580 \mathrm{~nm}$ and $420-850 \mathrm{~nm}$ to designate single features based on the wavelength kinetical behaviour. As observed before, the fast $\Delta A$ is centred around $535 \mathrm{~nm}$ (Fig. 11a). The relative cumulative $\Delta A$ change of this process, calculated for each wavelength in the region $510-570 \mathrm{~nm}$ during $t=0-3 \mathrm{~min}$, showed a logarithmic trend for all wavelengths (Fig. 11b). Different rates in $\Delta A$ per wavelength were observed, with $\lambda<535 \mathrm{~nm}$ developing $\Delta A$ at a slower rate than $\lambda \sim 560 \mathrm{~nm}$. Analysing the slower absorbance event with onset after 4 min of high light exposure, a smooth spectral feature in the visible and NIR region is revealed (Fig. 11c). Besides the previously shown local green 550$\mathrm{nm}$ absorbance peak, a large $\Delta A$ was manifested also in the 700-800 $\mathrm{nm}$ range, with a wide peak centred around $750 \mathrm{~nm}$. All wavelengths clearly developed at a similar kinetic rate in the entire $450-750 \mathrm{~nm}$ range, following a sigmoid function, confirming a single process is dynamically observed (Fig. 11d). This slow but significant

for $L$ in the VIS (400-620 nm) and for $F$ in the fluorescence range $(650-800 \mathrm{~nm})$, with the spectrum at $t=0 \mathrm{~min}$ taken as the reference spectrum (c, d). The wavelengths of the maximal $\Delta R$ and $\Delta T$ change within the 430-600 nm range during the transient are plotted in $\mathbf{e}$ and $\mathbf{f}$

process caused a $2 \%$ absorbance increase at the $550-\mathrm{nm}$ peak (similar to the quick feature at $535 \mathrm{~nm}$ ) and a $6-10 \%$ absorbance increase around $750 \mathrm{~nm}$. Finally, the absorbance profile during the 10-min transient showing the quick (480-580 nm in Fig. 10a, omitting the $F$ changes) and slow (400-800 $\mathrm{nm}$ in Fig. 10b) changes is provided in Fig. 12a. Several examinations of the experimental data revealed that the slow absorbance increase was typically observed from $t=5 \mathrm{~min}$ onward, but probably not always terminated at $t=10 \mathrm{~min}$ (Fig. 12b).

\section{Discussion}

The detection of spectrally contiguous radiance changes in the VIS-NIR range allowed us to detect, for the first time passively, specific leaf spectral behaviour in the absorbed photosynthetic active radiation (APAR) range with different onsets during strong light adaptation. In 


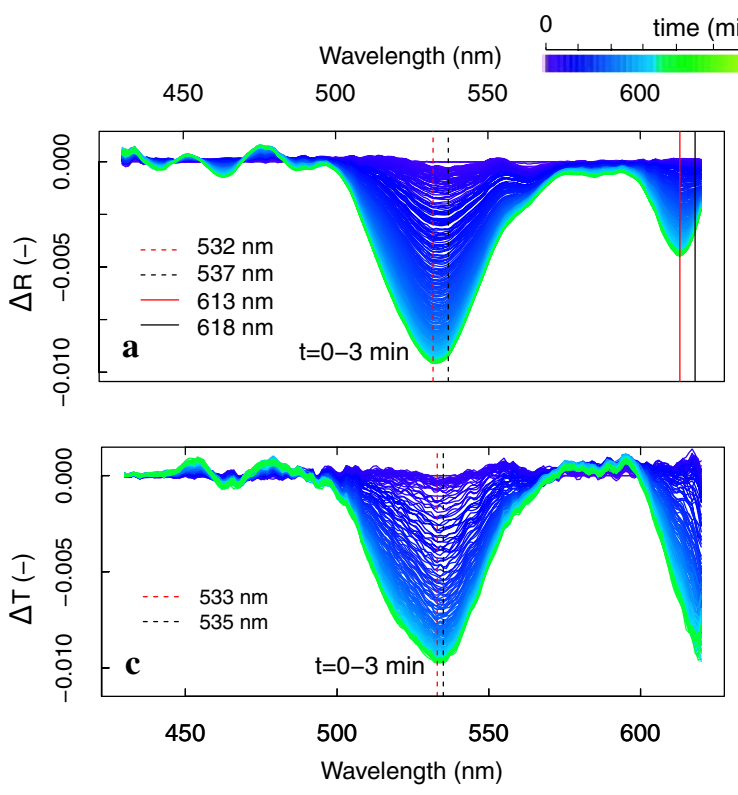

Fig. 8 Reflectance and transmittance change $(\Delta R$ and $\Delta T)$, separated into the fast $(t=0-3 \mathrm{~min})$ and slow $(t=3-10 \mathrm{~min})$ changes during photoprotection reactions of a dark-adapted $M$. alba leaf exposed to

contrast to the ultrafast transient absorption spectroscopy of isolated chloroplasts, pigments, or Lhc units that promote single molecules or complexes to an excited state, slower mechanisms are observed during the NPQ built-up phase. NPQ is a kinetically and mechanistically dynamic process, wherefore typically the short-term regulatory chemical and molecular mechanisms have been studied (Deamer et al. 1967; Heber 1969; Bassi and Caffarri 2000; Moya et al. 2001; Ruban et al. 2007, 2012; Johnson et al. 2009a; Johnson and Ruban 2014; Krüger et al. 2014b). It is well understood that two main quick NPQrelated absorption mechanisms occur in the short time upon a $\Delta \mathrm{pH}$, with structural rearrangements of the Lhcs as a result. One is the VAZ operation with a pigmentbased absorbance feature and the second is the quick PsbS-induced conformational changes or the detachment of the major antenna complexes from the PSII-LHCII core (Kramer and Sacksteder 1998; Johnson and Ruban 2014). In contrast, the occurrence of the slower induced, socalled energy-independent photoprotection mechanisms (Lambrev et al. 2012) are less understood from in vivo observations. Next, we discuss the observed spectral APAR changes and their relation to the NPQ processes, considering additional processes affecting the scattering behaviour at leaf surface as well.

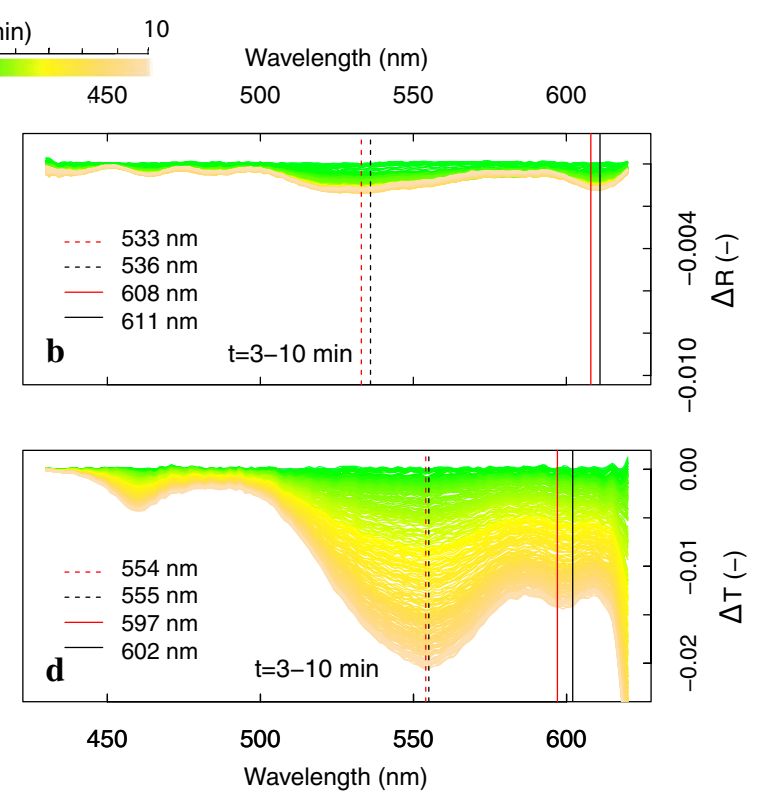

a high irradiance $\left(1311 \mu \mathrm{mol} \mathrm{m} \mathrm{m}^{-2} \mathrm{~s}^{-1}\right)$. Location of peak maxima are given for the start (black lines) and the end (red lines) of the transient phases

\section{Fast carotenoid conversions and membrane re-organizations}

Carotenoids are known to absorb light at similar wavelengths, with slight spectral shifts and peak positions, due to the conjugation length of the Car, the conformation of the pigment-protein complex (e.g. twisting) and the local environment (e.g. polarity of the medium) (Polívka and Sundström 2004; Polívka and Frank 2016). The S0-S2 transition of the different carotenoids is strongly allowed and visible as the three-peak absorption in the 400-500 $\mathrm{nm}$ range in solution. In vivo, protein-bound carotenoids show a red-shift in the absorption spectrum due to the dispersion interactions of the carotenoid with the protein (Polívka and Frank 2016). Due to this absorbance shift, the chemical conversion of the VAZ carotenoids in vivo is observed as an absorbance change in the 500-570 $\mathrm{nm}$ region with maximum around $531 \mathrm{~nm}$ (Gamon et al. 1990; Peñuelas et al. 1995). The linear correlation between the de-epoxidation state (DEPS) of the VAZ cycle and the reflectance decrease at $531 \mathrm{~nm}$ during the first minutes is well known from light transients on intact leaves, as indicated by an accumulation of both $\mathrm{A}$ and Z (Bilger et al. 1989; Gamon et al. 1990; Gamon and Surfus 1999; Peguero-Pina et al. 2013). The chemical VAZ conversion observed in this region is hereby exploited for computation of the Photochemical Reflectance Index, i.e. (R531-R570)/(R531 + R570) (Gamon et al. 1990, 1992; Peñuelas et al. 1995). Commonly used in remote sensing applications as indicator for the DEPS, PRI serves as a proxy 

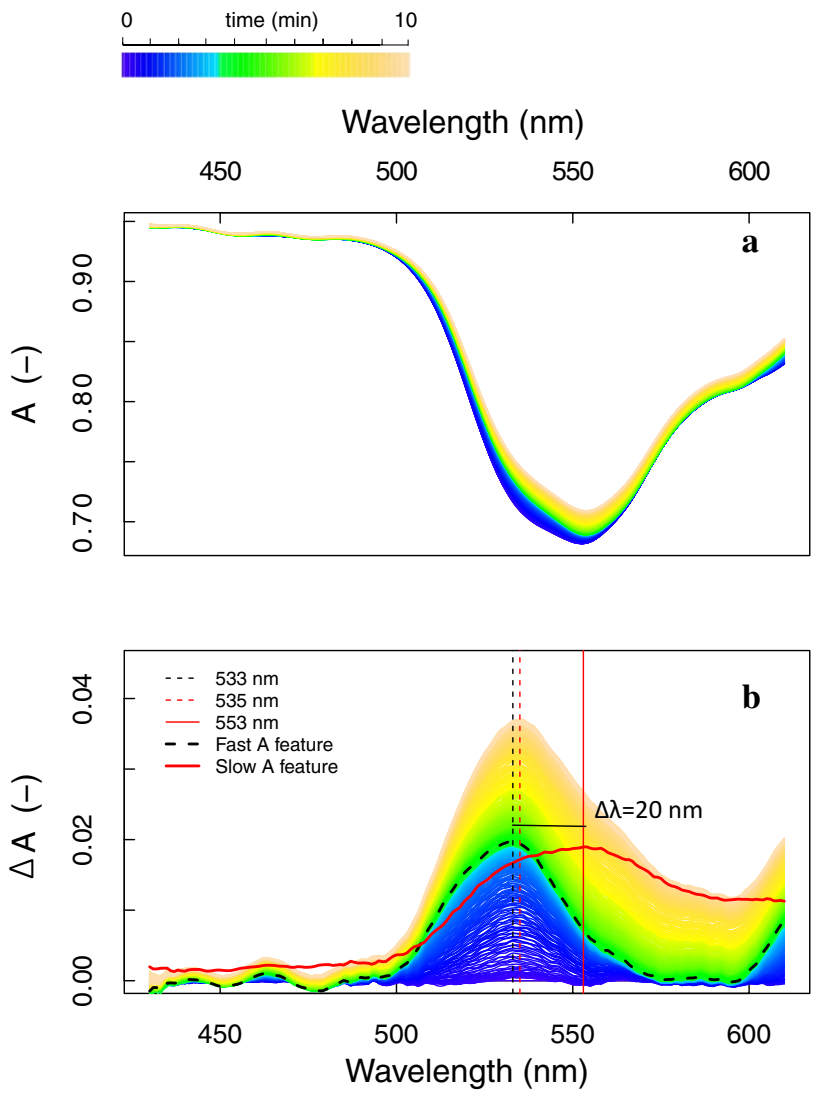

Fig. 9 Absorbance $(A,-)$ (a) and absorbance change $(\Delta A,-)$ (b) for a spectral interval of 430-620 nm during photoprotection of a dark-adapted $M$. alba leaf exposed to a high irradiance $\left(1311 \mu \mathrm{mol} \mathrm{m} \mathrm{m}^{-2} \mathrm{~s}^{-1}\right)$; The fast $(t=0-3 \mathrm{~min})$ and slow $(t=3-10 \mathrm{~min})$ absorbance features changes are displayed as the bold dashed black and the solid red line, respectively. The absorbance maxima at the start (black dashed) and at the end (red dashed) of the 10-min transient are plotted together with the absorbance peak of the slow component (red solid)

for the energy or $\Delta \mathrm{pH}$-dependent NPQ of vegetation at the leaf and, after required corrections, also at the canopy level. However, other scattering and absorbance shifts than those purely caused by the VAZ chemical conversion are described for the quick phase (Kramer and Crofts 1996; Johnson and Ruban 2014), which may interfere with the concept of a two-band Index (Van Wittenberghe et al. 2018). As a result, and due to the lack of normalization for the pigment pool (Ripullone et al. 2011), the functional link between PRI and DEPS is not always a straightforward relationship, even at the leaf level.

In the short term during strong $F$ quenching, we observed several phenomena in the 500-570 $\mathrm{nm}$ region with different onsets (Fig. $4 \mathrm{a}-\mathrm{h}$, dark grey area). In chronological order upon HL, the following events occurred: (1) an instantaneous two-peaked scattering decrease with maxima around 530 and $560 \mathrm{~nm}$ (Fig. 4a, b), followed by (2) a phase whereby $F$ is rapidly quenched without further spectral changes
(Fig. 4c, d), and next (3) the start of a more prominent feature with a peak around $531 \mathrm{~nm}$ (Fig. 4e, f). These observations confirm the $F$ quenching associated with the proton gradient (qE) involving two separate but interrelated phenomena: $\mathrm{qE}$-quenching itself ( $\Delta \mathrm{pH}$ built-up), and a modulation or amplification of the quenching by $\mathrm{A}$ and $\mathrm{Z}$ formation (Demmig et al. 1987). The instantaneous scattering in the same spectral region (Fig. 4a, b) is hypothesized as a low lumenal $\mathrm{pH}$-driven binding of free and available $\mathrm{Z}$, prior to a sufficient $\Delta \mathrm{pH}$ to activate the VAZ cycle. Similar to the first 2-peak feature in the 500-570 nm region, Kramer and Sacksteder (1998) observed an identical $\Delta A$ in a short time range of $450 \mathrm{~ms}$ upon sudden illumination of an intact leaf, followed by a further one-peak absorbance feature (500-570 nm) stabilizing after $20 \mathrm{~s}$. Compared to our measurements, their experiments reached a faster equilibrium of both absorbance changes, which might be attributed to the use of weaker illumination $\left(300 \mu \mathrm{mol} \mathrm{m}^{-2} \mathrm{~s}^{-1}\right)$, resulting in a smaller Car pool and faster equilibrium to dissipate the lower photon excess. Apart from this, we have not found any further similar shaped spectral evidence upon sudden illumination. Being located in the green spectral region where A and Z absorption appears in vivo (Gamon et al. 1990, 1992; Peñuelas et al. 1995; Gamon and Surfus 1999), a free and sustained $\mathrm{Z}$ pool is suggested to be available prior to illumination, binding to the complexes with the onset of lumen acidification. A sustained $\mathrm{Z}$ pool can act a membrane stabilizer in vivo in the lipid phase of the thylakoid membrane (Havaux 1998; Havaux and Niyogi 1999) being involved in antioxidant activity (Havaux and Niyogi 1999; Niyogi 1999; Dall'Osto et al. 2010). Low luminal pH, on the other hand, results in the protonation of specific antenna complexes (Dominici et al. 2002), which, synergistically with Z binding, leads to a conformational change (Moya et al. 2001; Dall'Osto et al. 2005, 2010). In addition, it has been recognized that Z-dependent sustained photoprotection, often grouped under the term qI, consists of two components, one sensitive and the other insensitive to the uncouplers, suggesting different mechanisms operating under different conditions and time scales (Ruban and Horton 1995; Dall'Osto et al. 2005; Nilkens et al. 2010; Ruban et al. 2012). As such, this spectral observation (Fig. 4a, b) could find an explanation in the binding of a readily available $\mathrm{Z}$ pool, before the proton gradient is sufficient to start the VAZ cycle. However, since several xanthophylls absorb in a similar region, this hypothesis would need to be further confirmed based on pigment analyses before and during a dark-to-HL transient protocol.

After a $F$ quenching devoted to the qE-quenching $(\Delta \mathrm{pH}$ built-up) itself (Fig. 4c, d), Car conversion (VAZ cycle) becomes visible in the $500-570 \mathrm{~nm}$ spectral region in the following minute, with further strong $F$ quenching (Fig. $4 \mathrm{~g}$, h). Bi-directional $\Delta L_{R}$ and $\Delta L_{T}$ measurements show a 


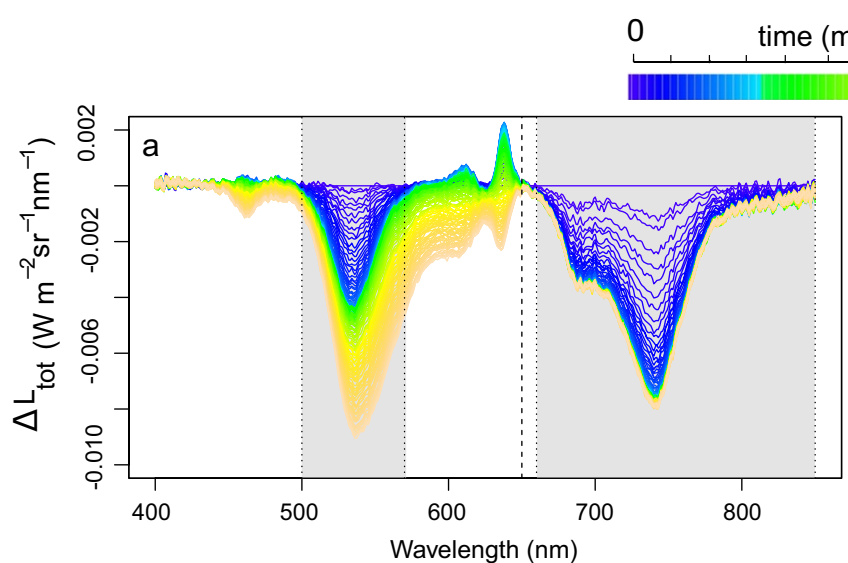

10
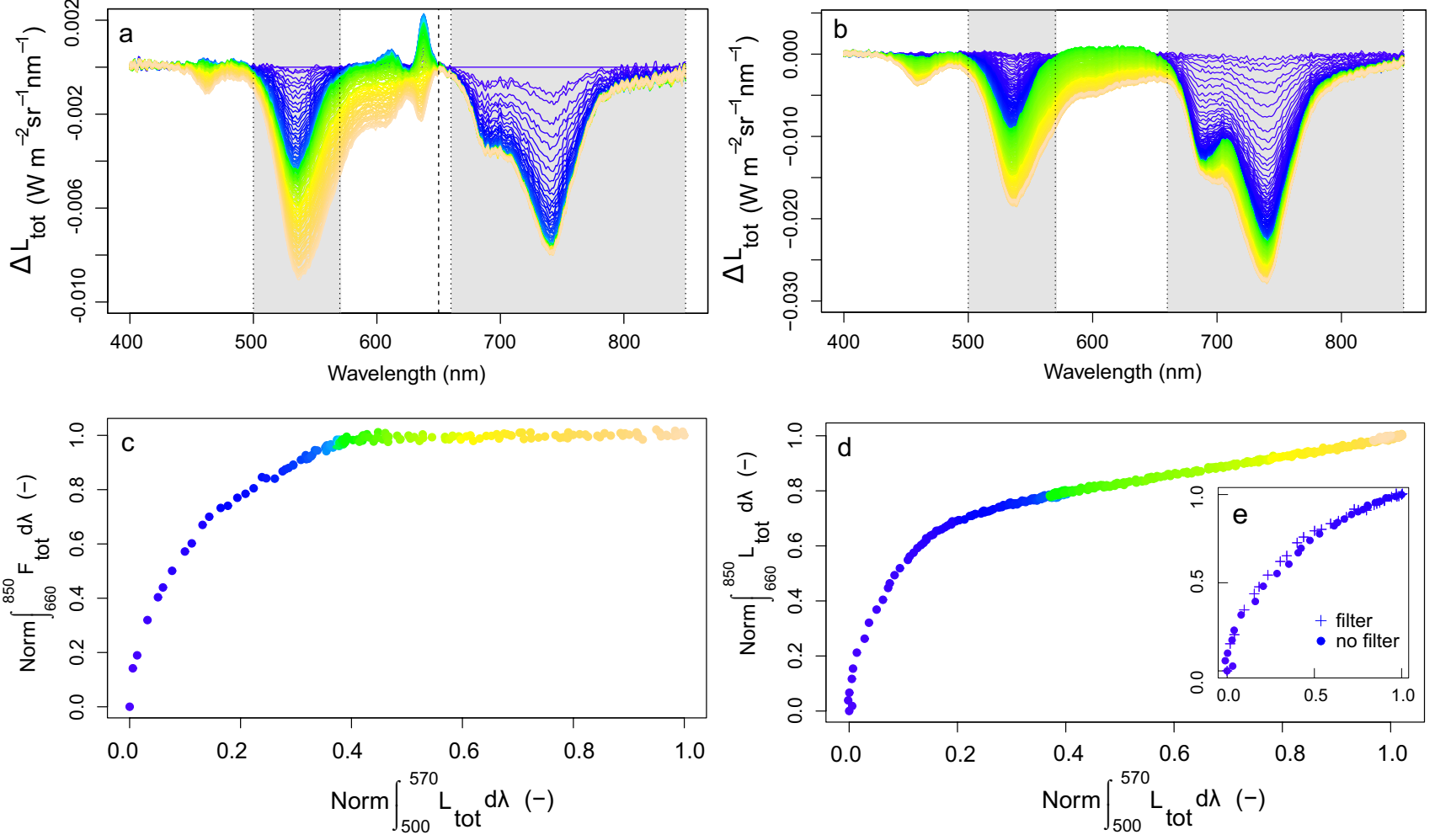

Fig. 10 Total radiance changes $\left(\Delta L_{\text {tot }}\right)$ for a J. regia leaf measured according protocol 1, i.e. with filter (a) and according protocol 2, i.e. without filter (b) the 650-nm cut-off filter during a 10-min dark-tohigh light transient. $\Delta L_{\text {tot }}$ values for the spectral ranges $500-570$ and $660-850 \mathrm{~nm}$ (marked as grey shades) were integrated and normal-

ized with the integral of the total change at the corresponding spectral ranges during $t=0-10 \mathrm{~min}$ with (c) and without (d) filter, and additionally for the period $t=0-2 \mathrm{~min}$, in which only fluorescence coupled radiance changes took place (e)

parallel and consistent decrease for all measurements, supporting a true absorbance increase at these wavelengths, expected for a chromophore conversion. In parallel to this consistent VAZ absorbance feature, co-occurring fast features are observed either manifesting a decreased (Fig. 4) or increased scattering behaviour with peaks located around 460, 615 and 645 (filter-affected) nm (Figs. 3a, 10a). The spectral signature of these changes are consistent with those observed from the light-induced reorganizations (unstacking) of the thylakoid membranes seen by circular dichroism spectroscopy (Garab 2014). These reversible changes in the organization of the thylakoid membranes occur on the time scale of seconds and minutes depicting the quick disassembly of the multilamellar structures (Garab 2014). Such fast structural response is given the fact that the electrostatic interactions between adjacent lamellae are generally weak (Garab et al. 1991) providing a generally low structural stability compared to e.g. the electrostatic interactions between the complexes themselves (Garab 2014). The physiological significance of this fast membrane flexibility is not fully understood yet, but unstacking or a light-induced increase

in the interthylakoidal space would facilitate the mobility of the complexes, required e.g. for the operation of the PSII repair mechanism (Yamamoto et al. 2008). A dip around $615 \mathrm{~nm}$ corresponding to the $\mathrm{Chl} \mathrm{Q}_{\mathrm{x}}$ state and decrease of the $675 \mathrm{~nm}$ region (here observed at $645 \mathrm{~nm}$ ) have been identified for quenched states of isolated membranes or isolated LHCII (Ruban et al. 2007). The transient development of these spectral features shown here, illustrating the structural flexibility of the thylakoid membranes in vivo, operates within the same time scale as the VAZ cycle. However, in contrast to the VAZ absorption, scattering changes due to membrane reorganization may not always occur (e.g. Fig. 10b), or show opposite scattering behaviour (Figs. 3left vs. 4), indicating both processes are not directly related to each other.

\section{Fluorescence quenching and re-absorption during fast pigment bed changes}

Major $F$ quenching is seen during the first 3-5 min upon strong illumination, when Car conversion and membrane 

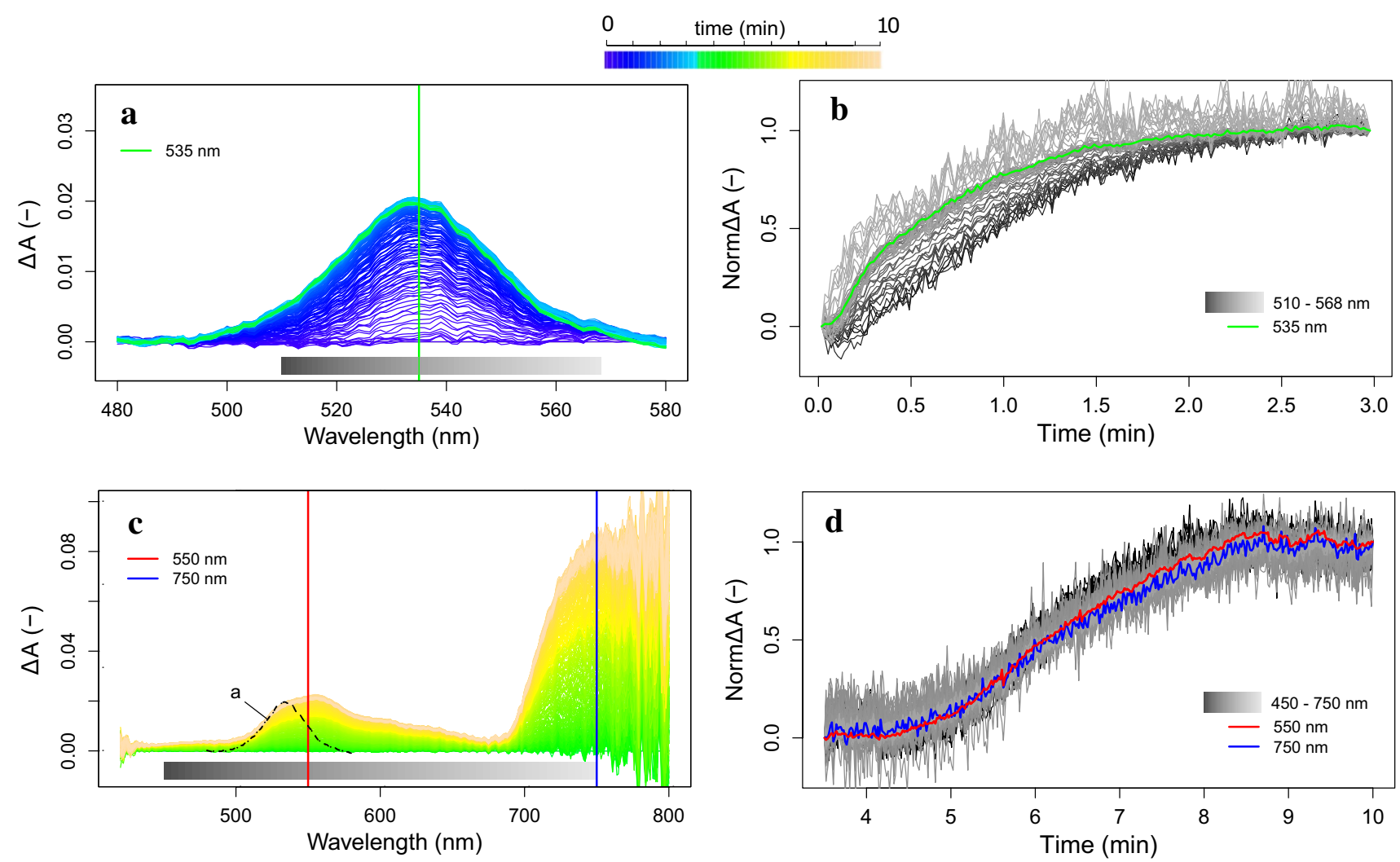

Fig. 11 Absorbance changes $(\Delta A)$ during a 10-min high-intensity illumination $\left(1283 \mu \mathrm{mol} \mathrm{m} \mathrm{m}^{-2} \mathrm{~s}^{-1}\right)$ exposure of a dark-adapted J. regia leaf, split between the fast ( $t=0-3 \mathrm{~min})$ Car conversion (a) and slow $(t=3-10 \mathrm{~min})$ conformational changes $(\mathbf{c})$, in both cases referenced by substraction of the absorbance spectrum at the beginning of each

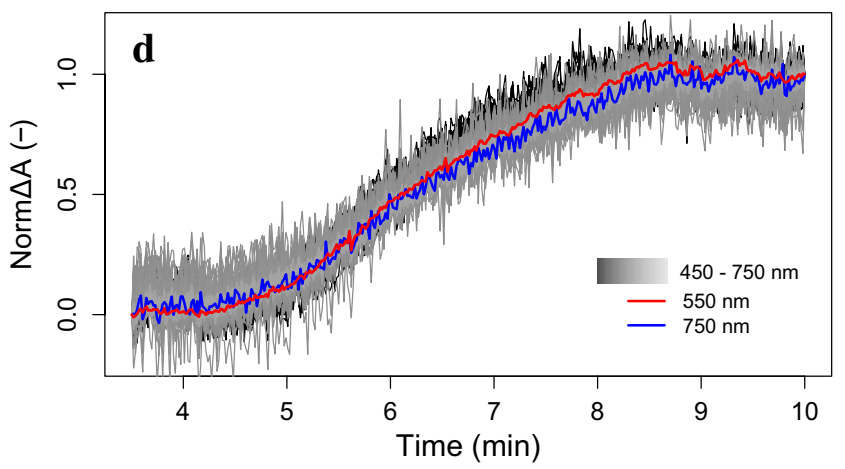

phase. The dynamic $\Delta A$ normalized for the total change of each phase is plotted in time for each phase and each wavelength (marked by grey scales in a and $\mathbf{c}$ ), representing the kinetical dynamic of each process $(\mathbf{b}, \mathbf{d})$. The evolution of the absorption change at the peak wavelengths in the VIS-NIR spectral range are given in colour

reorganizations were observed (Fig. 5). During $F$ quenching, we observed a small rapid increase in the F687/F740 ratio, followed by a stronger and general decrease the following minutes (Fig. 5d). Possible causes for a $F$ peak ratio here, are (i) a change in the relative share of PSI versus PSII $F$ emission i.e. due to short-term alternations of the relative PSIPSII antenna sizes, and/or (ii) an enhanced re-absorption of the red peak (F687) by Chl a, and/or (iii) new fluorescing components of the PSII antenna during NPQ (Miloslavina et al. 2008; Lambrev et al. 2010). Since state transitions between PSI and PSII, balancing light absorption when light quality changes (Niyogi 1999; Xu et al. 2015), are primarily linked to low light conditions, they are considered less important under HL. Lambrev et al. (2010) took a detailed investigation on the NPQ-associated spectral changes in the $F$ spectra of Arabidopsis (Arabidopsis thaliana) measured at room temperature and at $77 \mathrm{~K}$ and found for both setups a $F$ decrease in the red and a $F$ increase in the far-red range $(>710 \mathrm{~nm}$ ), with the first effect larger at room temperature. Mutant analysis showed moreover that the presence or absence of PsbS had a strong impact on the FR changes but not on the red changes, concluding these changes were

related to distinct NPQ processes. Interestingly, when Lhcs form aggregates, or higher orders oligomers, they exhibit a relatively enhanced $F$ in the far-red region (Miloslavina et al. 2008). Formation of a Chl-Chl charge transfer complex was suggested to be the underlaying cause of this differential $F$ quenching (Miloslavina et al. 2008). Formation of such complexes, as a result of $\mathrm{Chl}$ aggregation when two $\mathrm{Chl}$ molecules interact at a intermolecular separation less than a critical distance of $12 \AA$ (Beddard and Porter 1976), is suggested to play a role in $\mathrm{qE}$ and the source for the proposal of exclusively Chl-based quenching (Crofts and Yerkes 1994; Horton et al. 1996; Krüger and van Grondelle 2017). Here, we observed a significant increase in absorption at 615 and $645 \mathrm{~nm}$ (Fig. 4i, j) due to membrane reorganizations in the short term. They, however, do not appear as broad absorption bands as would be expected due to strong interpigment coupling for charge transfer states (Krüger and van Grondelle 2017). Their appearance in the red region (only observable until $650 \mathrm{~nm}$ due to the filter) could, however, explain an enhanced red $F$ re-absorption by $\mathrm{Chl}$ a causing the decrease of the F687/F740 ratio during quick arrangement of the membrane upon excessive light. 

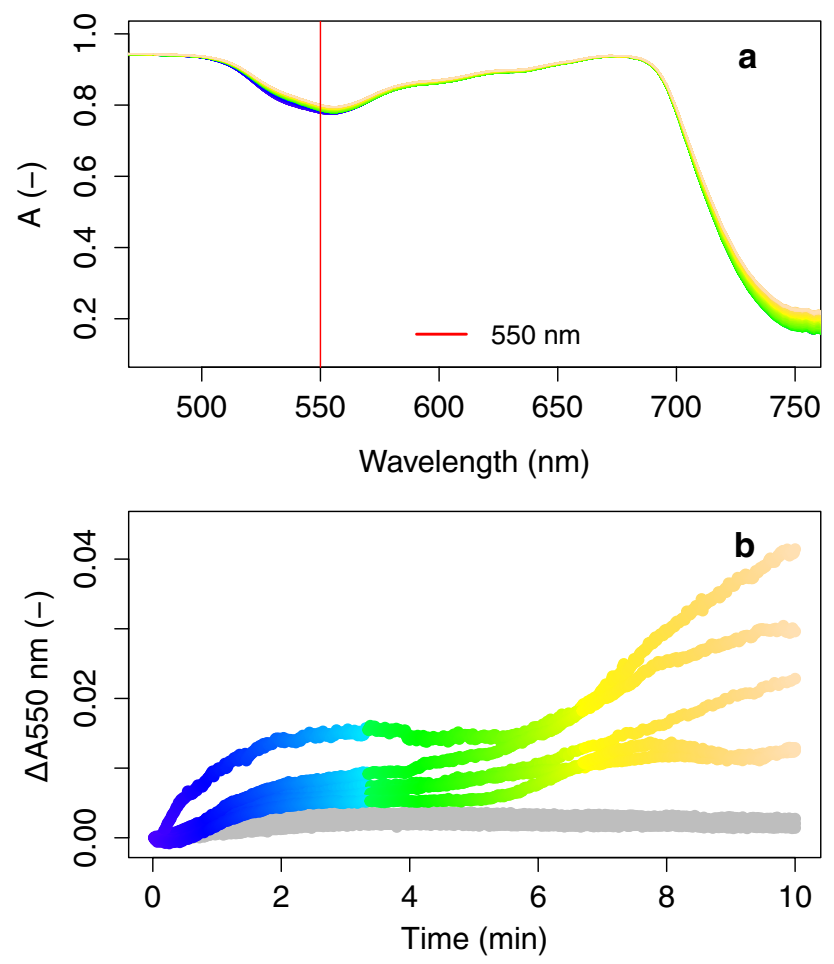

Fig. 12 Absorbance profile during the 10-min transient (by colour scale) with shifts caused by the energy-dependent chemical Car conversion (in blue colours) and the slow conformational switch (in green-to-yellow colours) (a) and $\Delta A$ at $550 \mathrm{~nm}$ for several leaf transient observations without (grey, $n=3$ ) and with (colour scale, $n=5$ ) the conformational switch (b)

\section{Slowly induced conformational pigment-protein changes in the VIS-NIR}

Following the fast carotenoid conversion and membrane reorganizations, a slower broad VIS-NIR absorbance increase was observed for both species, identified as one dynamical process (Fig. 11c, d). The action feature characterized by a consistent green and far-red absorbance increase intuitively contradicts with a light avoidance strategy. In what follows next, we intend to aggregate the arguments and provide an evidence-based framework, which help to understand the slow manifestation of this mechanism which has not been measured before in the view of light excess. Hereby, we discuss certain confusing phenomena (e.g. chloroplast motion) and instrumental artefacts that could be (mis)understood from the given observations:

i. Instrumental reliability and set-up Any instrumental or set-up-induced artefact constant in time which would alter the given radiance flux (Supplementary Material S1) is accounted for, diminished or would be nullified calculating dynamical spectral radiance flux changes. Dynamical artefacts such as possible instru- mental drift are not compatible or realistic with our observations (up to $8 \% L_{\text {tot }}$ signal change in the NIR within $10 \mathrm{~min}$ ), given the specific spectral nature and temporal behaviour of the feature. No LED instability was observed, verified by the surface radiance $L_{\mathrm{S}}$ before and after each transient, and would be seen as an overall (irregular) increase or decrease in $L_{\text {up }}$ or $L_{\mathrm{dw}}$, inconsistent with the spectral feature;

ii. Absorbance increase At the intact leaf level, in-path scattering changes are neutralized by the multiple scattering inside the leaf, wherefore, the $\Delta A$ is given only by specific absorbance changes. This allows us to observe specific spectral patterns for leaf-internal fast Car conversion, membrane reorganizations or slow chloroplast motion at a high temporal resolution. A decrease in both consistent forward and backscattered diffuse light during these slow observations indicates a true specific absorbance increase (Fig. 12a, b), whereby transmitted light changes are stronger affected (Figs. 7, 8). An overall absorbance increase contradicts to slow chloroplast motion, involving a spectrally broad $\Delta A$ decrease demonstrating a lightavoidance strategy (Deamer et al. 1967; Brugnoli and Björkman 1992; Dutta et al. 2017). The slow $\Delta A$ increase is further spectrally distinctive from chloroplast motion due to the lack of strong peak features in the 400-500 $\mathrm{nm}$ region (Fig. 11c) and the absence of any $F$ decrease (Fig. 10c). A specific absorbance increase also further contradicts to the absorbance changes in the VIS-NIR during progressive desiccation (water absorbs in the NIR), which would be manifested as an absorbance decrease in the NIR (Peñuelas and Inoue 1999);

iii. Light-harvesting antenna framework Given the broad nature and specific shape of the absorbance increase, any chemical change leading to such a new absorbance component is however not considered realistic. On the other hand, red-shifted spectra of Car and Chl absorptions as well as for $F$ bands have been commonly observed under high light exposure during $\mathrm{qE}$ formation of isolated Lhcs (Bode et al. 2009; Liao et al. 2010), or for Chl $F$ spectra (Johnson and Ruban 2009) and in vivo (Bode et al. 2009; Lambrev et al. 2010). The explanation for these absorbance shifts can be found in the fact that protein-embedded pigments are known to serve as effective probes of conformational changes providing structural flexibility, affecting their spectral properties (Scholes et al. 2011; Krüger and van Grondelle 2017). Hereby, the protein serves as the scaffold that controls the organization of the pigments in the Lhcs whereby strong intrapigment interactions can considerably increase the sensitivity of the pigments to the local environment 
(Novoderezhkin et al. 2007). In response to light excess, the local environment of the Lhcs is shown to be heterogeneous dielectric, profoundly tuning the energy transfer between pigments, caused by changes is the relative orientations of the pigments (Curutchet et al. 2011). The formation of the earlier mentioned red-shifted bands of Car and Chl has been correlated to the excitonic coupling between the S1 state of carotenoids and the lower states of Chl whereby, surprisingly, and increased $\mathrm{Car} \rightarrow \mathrm{Chl}$ as well as $\mathrm{Chl} \rightarrow \mathrm{Car}$ energy transfers were observed (Bode et al. 2009). Such interactions can occur when the two state energies are similar and there is as significant electronic coupling, which has been confirmed to apply for the Car S1 state and the lowest excited singlet states of Chl a, Chl $\mathrm{Q}_{\mathrm{y}}$ (Bode et al. 2009). Under these conditions, the coupling creates two new states a least partially delocalized over both pigments, leading to a collective excitation known as exciton. Exciton states seem to be present to some extent in all photosynthetic Lhcs (Scholes and Fleming 2006), and can shift spectra substantially, leading to quite marked spectral inhomogeneity (Müh et al. 2010). These observations have however only been observed in the fast response to light and linked to the PsbS-dependent conformational change during $\mathrm{qE}$;

iv. Timing The onset of the absorbance change takes place after 3-5 min of constant HL exposure, when the fast changes have terminated. This behavioural time pattern seen for all observations (Fig. 12b) suggests the spectral changes might relate to the preceding fast changes, e.g. the formation and binding of $\mathrm{Z}$, indirectly and invariably observed during the first minutes (Fig. 11a, b). The current acceptance of $\mathrm{Z}$ being the allosteric regulator for the slow conformational changes (Ruban et al. 2007; Ilioaia et al. 2011), might suggest the observed spectral changes relate to a structural modification induced after binding of $Z$ (see point vi), although further triggers would apparently be needed. At the Lhc level, Z-dependent quenching is observed as a more slowly induced reorganization compared to the fast PsbS-dependent conformational changes (Holzwarth et al. 2009; Nilkens et al. 2010; Lambrev et al. 2012). The latter were, however, not observed;

v. Kinetics The evolution of the spectral feature shows a slowly developing kinetic behaviour $(\Delta t=7 \mathrm{~min})$ with strong sigmoidicity (Fig. 11d). Sigmoidal kinetic profiles are the result of enzymes that demonstrate positive cooperative (allosteric) binding, i.e. binding of a ligand at one site of the macromolecule increasing the enzyme's affinity for another ligand at a site different from the other site (cf. Hill equation). During qE, such kinetical behaviour has been observed (Horton et al. 2000). For enzymatic reactions with multiple substrate-binding sites such as the Lhcs, this increased affinity for the substrate can cause a rapid and coordinated increase when a certain concentration of ligands is bound to the macromolecule. The protein-embedded pigments of the Lhcs not only interact with one another but also with the protein itself, and, hence, protein structural fluctuations (e.g. as a result of an enzymatic binding reaction) may significantly alter their spectroscopic and light-harvesting properties by tuning the pigment's transition energy (see point iii). Our observations showing the simultaneous spectral dynamic according to this slow sigmoid kinetic suggest the interaction between the protein scaffold and the pigments (see point vi) plays an important role. It should be mentioned, that our monitoring time was likely often too short to monitor the slowly induced conformational change being built-up until the final state (Fig. 12b), except the cases shown in Figs. 7-11 showing a steady-state or saturation in the signal response. Hence, a longer observation time is advisable. This was also recently corroborated by 20-min light transients performed on Fagus sylvatica L. leaves, showing identical strong slow absorbance increases, observable from the reflectance signal (Van Wittenberghe et al. 2018);

vi. Shape and peak locations The observed smooth absorbance change shows widened absorbance features around 550 and $750 \mathrm{~nm}$, which suggests involvement of both red-shifted Car and Chl (point iii). Other proteins or molecules may absorb in the NIR (e.g. plastocyanin, P700+), however there is no experimental evidence they show any (slow) absorbance dynamics upon NPQ induction. Figures $9 \mathrm{~b}$ and $11 \mathrm{c}$ illustrate for both $M$. alba and J. regia the similar magnitude (i.e. $2 \%$ ) in $\Delta A$ and shape between the VAZ feature and the slow feature, seemingly affected by a 20-nm spectral shift and very large band broadening. The conformational motions of pigment-protein complexes perturbing the site energies can be considered an explanation for these effects (see point iii). As mentioned, the energies of excitonic transitions are determined by the combined action of exciton shifts (causing homogeneous broadening of the main absorption peak together with the appearance of vibrational wings) and reorganization shifts (causing inhomogeneous broadening due to disorder) (Novoderezhkin et al. 2007; Krüger and van Grondelle 2017). The $550-\mathrm{nm}$ feature indeed shows a strong vibrational tail until $680 \mathrm{~nm}$. A most plausible explanation is that the antenna complexes where the $\mathrm{Z}$ was earlier bound are further involved in the slow structural transition, 
observed from (1) the shifted peak location, (2) the equal magnitude, and (3) the specific timing, as the slow absorbance changes were only seen after the VAZ cycle terminated (Fig. 12b). Interestingly, the feature extends to the NIR and kinetically couples with a wide peak at $750 \mathrm{~nm}$ with an approx. $50 \mathrm{~nm}$ half bandwidth (Fig. 11c). These observations are similar to the red-shifted behaviour observed during $\mathrm{qE}$, explained by exciton mixing between Car $\mathrm{S} 1$ state and the $\mathrm{Chl} \mathrm{Q}_{\mathrm{y}}$ states at sub-leaf level (point iii), suggesting a similar mechanism. Low-energy Chls, also called red forms, have been observed in quenched states of individual complexes (Miloslavina et al. 2008; Wientjes et al. 2012), shifting the $F$ emission spectra in vitro. Differences in red absorbance $>700 \mathrm{~nm}$ from circular dichroism spectra also illustrated these red forms (Morosinotto et al. 2003). Moreover, the spectral shift and large homogenous broadening of these red forms were explained due to mixing with a CT state (Romero et al. 2009). Our in vivo observation of a very broad continuous absorbance feature over these specific wavelengths may not necessarily related to the same absorbance shift described during $\mathrm{qE}$ or PsbS-dependent conformational changes, but seem to indicate an analogous behaviour of strong coupling between the Car (suggestively Z) and Chl $\mathrm{Q}_{\mathrm{y}}$ states.

In vivo, regulated photoprotection has been commonly understood from quick $1 \mathrm{Chl} *$ excitation energy quenching or the $F$ quenching pathway only (cf. PAM-NPQ). However, the consensus is that non-photochemical quenching involves protein conformational changes, in the case of the fast changes, re-distributing the energy levels of particular pigments in the Lhcs and creating energy traps (Krüger and van Grondelle 2017). Two types of energy traps are under the loop: (1) a low-lying Car S1 state and, (2) a CT state either formed by Chl-Chl or Chl-Car interaction (Krüger and van Grondelle 2017). Experimental evidence for these formations has been given mostly by in vitro observations at sub-leaf level. However, at the intact leaf level specific absorbance changes resulting from the whole ensemble of internal structural rearrangements allows us to observe specific spectral dynamics and their dynamical and sequential behaviour. The strong absorbance increase observed according a slow sigmoid dynamic is hereby suggested to find its origin similar as the one of the known fast low-energy redshifted components in the absorption spectra of PSII peripheral antenna complexes during short-term fast conformational changes (Ruban et al. 1993; Miloslavina et al. 2008; Bode et al. 2009; Johnson et al. 2009a, b), suggesting these pathways for energy quenching. Hence, a low-energy (longwavelength) shifted feature indicating a strong coupling between Car (peak at $550 \mathrm{~nm}$ ) and Chl (peak at $750 \mathrm{~nm}$ ) states is proposed as possible explanation for our observations. Fast broadband shifted features during $\mathrm{qE}$ were not observed for the two species, indicating the absence of these PsbS-dependent structural changes. This could be considered in agreement with the models suggesting two independent NPQ sites (peripherical and core antenna) operating independently and allowing a fast and a slow regulation response to HL in order to avoid severe overshoots as well as undershoots (Holzwarth et al. 2009; Holzwarth and Jahns 2014). Moreover, although being tested under equal illumination and temperature conditions, the slowly induced absorbance shift did not take place systematically in all leaves tested after the occurrence of $\mathrm{Z}$ formation (Fig. 12b). A possible explanation we seek in this context is that the activation energy, required to arrive to this further state, is quite high. Protein motion can be described as transitions between different conformational sub-states, whereby the transition of the protein complex requires additional energy to cross barriers in the context of the conformational-energy landscape model (Krüger et al. 2014a). Hereby, the transition energies of the pigments and shapes of their absorption and fluorescence spectra, together with how the molecules are assembled, decide the energy landscape (Scholes et al. 2011). Depending on the pigment pool available and pigment bed organization formed by previous light conditions (even after $2 \mathrm{~h}$ darkness), the amount of energy needed to activate a further conformational change might have possibly varied between leaf samples. This probably makes the switch towards a further conformational state in the chain of conformational disorder within a short transient time of $10 \mathrm{~min}$ a relatively unpredictable event. Further experimental evidence from systematic experiments is needed to confirm this order of events for more species and to investigate the link between available pigment pools and the absorption dynamics upon light excess. Careful selection of species and growing conditions and the possible use of specific mutant species may help to further disentangle the absorbance dynamics related to the regulated and structure-based energy distribution and possible energy trapping of light excess.

\section{Conclusions}

Spectral dynamics of bi-directional diffusively scattered light at the leaf level upon illumination excess allow us to observe in vivo a complexity of structural adjustments at different hierarchical levels of the photosynthetic machinery. Fast dynamics involve a consistent VAZ conversion feature, often in parallel with more irregular membrane re-organizations within the first minutes, when $1 \mathrm{Chl} *$ or $F$ quenching takes place. VAZ conversion is hereby seen as an absorbance increase in the $500-570 \mathrm{~nm}$ region from both leaf sides due to a change in chromophore composition. As 
a slower response, a further and major VIS-NIR absorbance feature is observed and argued to be a shift due to a slow-induced pigment-protein conformational switch, with no $F$ quenching involved. This spectral feature is clearly distinct from the chloroplast motion photo-avoidance strategy that happens also slowly but is manifested as an overall absorbance decrease. The broadened and very significant green-red $(\sim 550 \mathrm{~nm})$ and near-infrared $(\sim 750 \mathrm{~nm})$ peaks in this smooth feature are suggested to be a low-energy shifted re-distribution of absorption bands of, respectively, Car and Chl, showing a strong exciton coupling. The biophysical cause of these changes is further argued to be found in the pigment-protein dynamics whereby spectral properties of the pigment bed are fine-tuned due to modified interactions between the chromophores. Yet, these new insights need to be corroborated by further well-designed experiments to confirm the observation of non-photochemical energy quenching identified as a structure-based energy dissipation mechanism detectable in vivo, over the full PAR spectrum and beyond $700 \mathrm{~nm}$. Certainly, the identification and unravelling of the dynamical spectral features detected through intact leaf spectroscopy seems to be a very promising direction in the further understanding of pigment-protein complex dynamics in their native environment. Such developments will allow us to observe the APAR energy distribution at a higher detail, i.e. with a harvesting or a dissipation fate, and to improve the monitoring of in vivo plant functioning from remote VIS-NIR hyperspectral observations.

Acknowledgements Open access funding provided by University of Helsinki. The work presented was funded by the European Union's H2020 Marie Skłodowska-Curie individual fellowship of the first author under the Grant Agreement FLUOPHOT - No [701815]. SVW also acknowledges her current postdoctoral scholarship APOSTD/2018/162 funded by the Generalitat Valenciana and cofunded by the 'Fondo Social Europeo'. Contribution of ZM was supported by the Australian Research Council Future Fellowship 'Bridging scales in remote sensing of vegetation stress' No [FT160100477].

\section{Compliance with ethical standards}

Conflicts of interest The authors declare that they have no conflict of interest.

Open Access This article is distributed under the terms of the Creative Commons Attribution 4.0 International License (http://creativeco mmons.org/licenses/by/4.0/), which permits unrestricted use, distribution, and reproduction in any medium, provided you give appropriate credit to the original author(s) and the source, provide a link to the Creative Commons license, and indicate if changes were made.

\section{References}

Aasen H, Van Wittenberghe S, Sabater Medina N et al (2019) Sun-induced chlorophyll fluorescence II: review of passive measurement setups, protocols, and their application at the leaf to canopy level. Remote Sens 11:927. https://doi.org/10.3390/ rs 11080927

Ahn TK, Avenson TJ, Ballottari M et al (2008) Architecture of a charge-transfer state regulating light harvesting in a plant antenna protein. Science 320:794-797

Alonso L, Gómez-Chova L, Vila-Francés, J., Amorós-López, J., Guanter, L., Calpe J, Moreno J (2007) Sensitivity analysis of the Fraunhofer line discrimination method for the measurement of chlorophyll fluorescence using a field spectroradiometer. In: Proceedings of the 3th international workshop on remote sensing of vegetation fluorescence. Florence, pp 3756-3759

Alonso L, Van Wittenberghe S, Amorós-López J et al (2017) Diurnal cycle relationships between passive fluorescence, PRI and NPQ of vegetation in a controlled stress experiment. Remote Sens 9:1-16. https://doi.org/10.3390/rs9080770

Bassi R, Caffarri S (2000) Lhc proteins and the regulation of photosynthetic light harvesting function by xanthophylls. Photosynth Res 64:243-256. https://doi.org/10.1023/A:1006409506272

Beddard GS, Porter G (1976) Concentration quenching in chlorophyll. Nature 260:366-367

Betterle N, Ballottari M, Zorzan S et al (2009) Light-induced dissociation of an antenna hetero-oligomer is needed for non-photochemical quenching induction. J Biol Chem 284:15255-15266. https ://doi.org/10.1074/jbc.M808625200

Bilger W, Björkman O (1990) Role of the xanthophyll cycle in photoprotection elucidated by measurements of light-induced absorbance changes, fluorescence and photosynthesis in leaves of Hedera canariensis. Photosynth Res 25:173-185. https://doi.org/10.1007/ bf00033159

Bilger W, Bjorkman O, Thayer SS (1989) Light-induced spectral absorbance changes in relation to photosynthesis and the epoxidation state of xanthophyll cycle components in cotton leaves. Plant Physiol 91:542-551

Bode S, Quentmeier CC, Liao P-N et al (2009) On the regulation of photosynthesis by excitonic interactions between carotenoids and chlorophylls. Proc Natl Acad Sci USA 106:12311-12316. https://doi.org/10.1073/pnas.0903536106

Brugnoli E, Björkman O (1992) Chloroplast movements in leaves: influence on chlorophyll fluorescence and measurements of light-induced absorbance changes related to $\Delta \mathrm{pH}$ and zeaxanthin formation. Photosynth Res 32:23-35. https://doi. org/10.1007/BF00028795

Buschmann C, Langsdorf G, Lichtenthaler HK (2000) Imaging of the blue, green, and red fluorescence emission of plant: an overview. Photosynthetica 38:483-491

Cazzaniga S, Dall'Osto L, Kong SG et al (2013) Interaction between avoidance of photon absorption, excess energy dissipation and zeaxanthin synthesis against photooxidative stress in Arabidopsis. Plant J 76:568-579. https://doi.org/10.1111/tpj.12314

Correa-Galvis V, Poschmann G, Melzer M et al (2016) PsbS interactions involved in the activation of energy dissipation in Arabidopsis. Nat Plants 2:1-8. https://doi.org/10.1038/NPLAN TS.2015.225

Croce R, Weiss S, Bassi R (1999) Carotenoid-binding sites of the major light-harvesting complex II of higher plants. J Biol Chem 274:29613-29623. https://doi.org/10.1074/jbc.274.42.29613

Crofts AR, Yerkes CT (1994) A molecular mechanism for qE-quenching. FEBS Lett 352:265-270. https://doi. org/10.1016/0014-5793(94)00976-7

Cseh Z, Vianelli A, Rajagopal S et al (2005) Thermo-optically induced reorganizations in the main light harvesting antenna of plants. I. Non-Arrhenius type of temperature dependence and linear light-intensity dependencies. Photosynth Res 86:263273. https://doi.org/10.1007/s11120-005-5104-1 
curutchet c, kongsted j, muñoz-losa a et al (2011) photosynthetic light-harvesting is tuned by the heterogeneous polarizable environment of the protein. J Am Chem Soc 133:3078-3084. https ://doi.org/10.1021/ja110053y

D'Haese D, Vandermeiren K, Caubergs RJ et al (2004) Non-photochemical quenching kinetics during the dark to light transition in relation to the formation of antheraxanthin and zeaxanthin. J Theor Biol 227:175-186. https://doi.org/10.1016/j. jtbi.2003.10.011

Dall'Osto L, Caffarri S, Bassi R (2005) A mechanism of nonphotochemical energy dissipation, independent from PsbS, revealed by a conformational change in the antenna protein CP26. Plant Cell 17:1217-1232. https://doi.org/10.1105/tpc.104.030601

Dall'Osto L, Cazzaniga S, Havaux M, Bassi R (2010) Enhanced photoprotection by protein-bound vs free xanthophyll pools: a comparative analysis of chlorophyll $b$ and xanthophyll biosynthesis mutants. Mol Plant 3:576-593. https://doi.org/10.1093/ $\mathrm{mp} / \mathrm{ssp} 117$

Deamer DW, Crofts AR, Packer L (1967) Mechanisms of light-induced structural changes in chloroplasts I. Light-scattering increments and ultrastructural changes mediated by proton transport. Biochim Biophys Acta 131:81-96. https://doi.org/10.1016/00052728(67)90032-1

Demmig B, Winter K, Krüger A, Czygan F-C (1987) Photoinhibition and zeaxanthin formation in intact leaves: a possible role of the xanthophyll cycle in the dissipation of excess light energy. Plant Physiol 84:218-224. https://doi.org/10.1104/pp.84.2.218

Demmig-Adams B, Adams WW III (1992) Photoprotection and other responses of plants to high light stress. Annu Rev Plant Biol 43:599-626. https://doi.org/10.1146/annurev.pp.43.06019 2.003123

Demmig-Adams B, Adams WW III, Logan BA, Verhoeven AS (1995) Xanthophyll cycle-dependent energy dissipation and flexible photosystem II efficiency in plants acclimated to light stress. Aust J Plant Physiol 22:249-260. https://doi.org/10.1071/ PP9950249

Dominici P, Caffarri S, Armenante F et al (2002) Biochemical properties of the PsbS subunit of photosystem II either purified from chloroplast or recombinant*. J Biol Chem 277:22750-22758. https://doi.org/10.1074/jbc.M200604200

Dutta S, Cruz JA, Imran SM et al (2017) Variations in chloroplast movement and chlorophyll fluorescence among chloroplast division mutants under light stress. J Exp Bot 68:3541-3555. https:// doi.org/10.1093/jxb/erx203

Evain S, Flexas J, Moya I (2004) A new instrument for passive remote sensing: 2. Measurement of leaf and canopy reflectance changes at $531 \mathrm{~nm}$ and their relationship with photosynthesis and chlorophyll fluorescence. Remote Sens Environ 91:175-185. https://doi. org/10.1016/j.rse.2004.03.012

Gamon JA, Surfus JS (1999) Assessing leaf pigment content and activity with a reflectometer. New Phytol 143:105-117. https://doi.org /10.1046/j.1469-8137.1999.00424.x

Gamon JA, Field CB, Bilger W, Björkman O et al (1990) Remote sensing of the xanthophyll cycle and chlorophyll fluorescence in sunflower leaves and canopies. Oecologia 85:1-7

Gamon JA, Peñuelas J, Field CB (1992) A narrow-waveband spectral index that tracks diurnal changes in photosynthetic efficiency. Remote Sens Env 44:35-44. https://doi.org/10.1016/00344257(92)90059-S

Garab G (2014) Hierarchical organization and structural flexibility of thylakoid membranes. Biochim Biophys Acta Bioenerg 1837:481494. https://doi.org/10.1016/j.bbabio.2013.12.003

Garab G (2016) Structural changes and non-photochemical quenching of chlorophyll a fluorescence in oxygenic photosynthetic organisms. In: Demmig-Adams B, Adams WW, Garab G (eds) Nonphotochemical quenching and energy dissipation in plants, algae and cyanobacteria. Springer, New York, pp 245-258. https://doi. org/10.1007/978-94-017-9032-1

Garab G, Kieleczawa J, Sutherland JC et al (1991) Organization of pigment-protein complexes into macrodomains in the thylakoid membranes of wild-type and chlorophyll b-less mutant of Barley as revealed by circular dichroism. Photochem Photobiol 54:273281. https://doi.org/10.1111/j.1751-1097.1991.tb02016.x

Havaux M (1998) Carotenoids as membrane stabilizers in chloroplasts. Trends Plant Sci 3:147-151. https://doi.org/10.1016/s1360 -1385(98)01200-X

Havaux M, Niyogi KK (1999) The violaxanthin cycle protects plants from photooxidative damage by more than one mechanism. Proc Natl Acad Sci USA 96:8762-8767. https://doi.org/10.1073/ pnas.96.15.8762

Heber U (1969) Conformational changes of chloroplasts induced by illumination of leaves in vivo. Biochim Biophys Acta 180:302319. https://doi.org/10.1016/0005-2728(69)90116-9

Heirwegh KPM, Meuwissen JATP, Lontie R (1987) Selective absorption and scattering of light by solutions of macromolecules and by particulate suspensions. J Biochem Biophys Methods 14:303-322. https://doi.org/10.1016/0165-022X(87)90025-X

Heyde S, Jahns P (1998) The kinetics of zeaxanthin formation is retarded by dicyclohexylcarbodiimide. Plant Physiol 117:659665. https://doi.org/10.1104/pp.117.2.659

Holt NE, Fleming GR, Niyogi KK (2004) Toward an understanding of the mechanism of nonphotochemical quenching in green plants. Biochemistry 43:8281-8289. https://doi.org/10.1021/bi0494020

Holzwarth AR, Jahns P (2014) Non-photochemical quenching mechanisms in intact organisms as derived from ultrafast-fluorescence kinetic studies. In: Demmig-Adams B, Adams WW, Garab G (eds) Non-photochemical quenching and energy dissipation in plants, algae and cyanobacteria. Springer, New York, pp 129-156. https ://doi.org/10.1007/978-94-017-9032-1

Holzwarth AR, Miloslavina Y, Nilkens M, Jahns P (2009) Identification of two quenching sites active in the regulation of photosynthetic light-harvesting studied by time-resolved fluorescence. Chem Phys Lett 483:262-267. https://doi.org/10.1016/j.cplet t.2009.10.085

Horton P, Ruban AV, Walters RG (1996) Regulation of light harvesting in green plants. Annu Rev Plant Physiol Plant Mol Biol 47:655684. https://doi.org/10.1146/annurev.arplant.47.1.655

Horton P, Ruban AV, Wentworth M (2000) Allosteric regulation of the light-harvesting system of photosystem II. Philos Trans Biol Sci 355:1361-1370. https://doi.org/10.1098/rstb.2000.0698

Ilioaia C, Johnson M, Liao P-N et al (2011) Photoprotection in plants involves a change in lutein 1 binding domain in the major lightharvesting complex of photosystem II. J Biol Chem. https://doi. org/10.1074/jbc.m111.234617

Inoue Y, Shibata K (1973) Light-induced chloroplast rearrangements and their action spectra as measured by absorption spectrophotometry. Planta 114:341-358. https://doi.org/10.1007/BF003 87947

Jahns P, Holzwarth AR (2012) The role of the xanthophyll cycle and of lutein in photoprotection of photosystem II. Biochim Biophys Acta Bioenerg 1817:182-193. https://doi.org/10.1016/j.bbabi o.2011.04.012

Johnson MP, Ruban AV (2009) Photoprotective energy dissipation in higher plants involves alteration of the excited state energy of the emitting chlorophyll (s) in the light harvesting antenna II (LHCII). J Biol Chem 284:23592-23601. https://doi.org/10.1074/ jbc.M109.013557

Johnson MP, Ruban AV (2014) Rethinking the existence of a steadystate delta pfi component of the proton motive force across plant thylakoid membranes. Photosynth Res 119:233-242. https://doi. org/10.1007/s11120-013-9817-2 
Johnson MP, Pérez-Bueno ML, Zia A et al (2009a) The zeaxanthinindependent and zeaxanthin-dependent $\mathrm{qE}$ components of nonphotochemical quenching involve common conformational changes within the photosystem II antenna in Arabidopsis. Plant Physiol 149:1061-1075. https://doi.org/10.1104/pp.108.129957

Johnson MP, Ruban AV, Johnson MP, Ruban AV (2009b) Photoprotective energy dissipation in higher plants involves alteration of the excited state energy of the emitting chlorophyll(s) in the light harvesting antenna II (LHCII). J Biol Chem 284:23592-23601. https://doi.org/10.1074/jbc.M109.013557

Kramer DM, Crofts AR (1996) Measurement of photosynthetic electron transport. In: Baker NR (ed) Photosynthesis and the environment. Kluwer Academic Publishers, New York, pp 25-66. https:// doi.org/10.1007/0-306-48135-9

Kramer DM, Sacksteder CA (1998) A diffused-optics flash kinetic spectrophotometer (DOFS) for measurements of absorbance changes in intact plants in the steady-state. Photosynth Res 56:103-112. https://doi.org/10.1023/A:1005968211506

Krause GH (1973) The high-energy state of the thylakoid system as indicated by chlorophyll fluorescence and chloroplast shrinkage. Biochim Biophys Acta 292:715-728. https://doi. org/10.1016/0005-2728(73)90019-4

Krüger TPJ, van Grondelle R (2017) The role of energy losses in photosynthetic light harvesting. J Phys B 50:132001

Krüger TPJ, Wientjes E, Croce R, Van Grondelle R (2011) Conformational switching explains the intrinsic multifunctionality of plant light-harvesting complexes. Proc Natl Acad Sci USA 108:1351613521. https://doi.org/10.1073/pnas.1105411108

Krüger TPJ, Ilioaia C, Horton P et al (2014a) How protein disorder controls non-photochemical fluorescence quenching. In: Demmig-Adams B, Garab G, Adams WW (eds) Non-photochemical quenching and energy dissipation in plants, algae and cyanobacteria. Springer, New York, pp 157-180. https://doi. org/10.1007/978-94-017-9032-1

Krüger TPJ, Ilioaia C, Johnson MP et al (2014b) Disentangling the low-energy states of the major light-harvesting complex of plants and their role in photoprotection. Biochim Biophys Acta Bioenerg 1837:1027-1038. https://doi.org/10.1016/j.bbabio.2014.02.014

Kumar R, Silva L (1973) Light ray tracing through a leaf cross section. Appl Opt 12:2950-2954

Lambrev PH, Nilkens M, Miloslavina Y et al (2010) Kinetic and spectral resolution of multiple nonphotochemical quenching components in Arabidopsis leaves. Plant Physiol 152:1611-1624. https ://doi.org/10.1104/pp.109.148213

Lambrev PH, Miloslavina Y, Jahns P, Holzwarth AR (2012) On the relationship between non-photochemical quenching and photoprotection of Photosystem II. Biochim Biophys Acta Bioenerg 1817:760-769. https://doi.org/10.1016/j.bbabio.2012.02.002

Latimer P, Pyle B (1972) Light scattering at various angles. Biophys J 12:764-773

Latowski D, Burda K, Strzałka K (2000) A mathematical model describing kinetics of conversion of violaxanthin to zeaxanthin via intermediate antheraxanthin by the xanthophyll cycle enzyme violaxanthin de-epoxidase. J Theor Biol 206:507-514. https://doi. org/10.1006/jtbi.2000.2141

Liao PN, Holleboom CP, Wilk L et al (2010) Correlation of car $\mathrm{S} 1 \rightarrow \mathrm{Chl}$ with $\mathrm{Chl} \rightarrow \mathrm{Car} \mathrm{S} 1$ energy transfer supports the excitonic model in quenched light harvesting complex II. J Phys Chem B 114:15650-15655. https://doi.org/10.1021/jp1034163

Miloslavina Y, Wehner A, Lambrev PH et al (2008) Far-red fluorescence: a direct spectroscopic marker for LHCII oligomer formation in non-photochemical quenching. FEBS Lett 582:3625-3631. https://doi.org/10.1016/j.febslet.2008.09.044

Morosinotto T, Breton J, Bassi R, Croce R (2003) The nature of a chlorophyll ligand in Lhca proteins determines the far-red fluorescence emission typical of photosystem I. J Biol Chem 278:49223-49229. https://doi.org/10.1074/jbc.M309203200

Moya I, Silvestri M, Vallon O et al (2001) Time-resolved fluorescence analysis of the Photosystem II antenna proteins in detergent micelles and liposomes. Biochemistry 40:12552-12561

Müh F, Madjet MEA, Renger T (2010) Structure-based identification of energy sinks in plant light-harvesting complex II. J Phys Chem B 114:13517-13535. https://doi.org/10.1021/jp106323e

Müller MG, Lambrev P, Reus M et al (2010) Singlet energy dissipation in the photosystem II light-harvesting complex does not involve energy transfer to carotenoids. ChemPhysChem 11:1289-1296. https://doi.org/10.1002/cphc.200900852

Myneni RB, Ross J (1991) Photon-vegetation interactions: applications in optical remote sensing and plant ecology. Springer, New York, p 565

Nilkens M, Kress E, Lambrev P et al (2010) Identification of a slowly inducible zeaxanthin-dependent component of non-photochemical quenching of chlorophyll fluorescence generated under steadystate conditions in Arabidopsis. Biochim Biophys Acta Bioenerg 1797:466-475. https://doi.org/10.1016/j.bbabio.2010.01.001

Niyogi KK (1999) Photoprotection revisited: genetic and molecular approaches. Annu Rev Plant Physiol Plant Mol Biol 50:333-359

Novoderezhkin VI, Dekker JP, van Grondelle R (2007) Mixing of exciton and charge-transfer states in photosystem II reaction centers: modeling of stark spectra with modified redfield theory. Biophys J 93:1293-1311. https://doi.org/10.1529/biophysj.106.096867

Ostroumov EE, Khan YR, Scholes GD, Govindjee (2014) Photophysics of photosynthetic pigment-protein complexes. In: DemmigAdams B, Garab G, Adams IWW (eds) Non-photochemical quenching and energy dissipation in plants, 97 algae and cyanobacteria. Springer, New York, pp 97-128

Pascal AA, Liu Z, Broess K et al (2005) Molecular basis of photoprotection and control of photosynthetic light-harvesting. Nature 436:134-137. https://doi.org/10.1038/nature03795

Peguero-Pina JJ, Gil-Pelegrín E, Morales F (2013) Three pools of zeaxanthin in Quercus coccifera leaves during light transitions with different roles in rapidly reversible photoprotective energy dissipation and photoprotection. J Exp Bot 64:1649-1661. https ://doi.org/10.1093/jxb/ert024

Peñuelas J, Inoue Y (1999) Reflectance indices indicative of changes in water and pigment contents of peanut and wheat leaves. Photosynthetica $36: 355-360$

Peñuelas J, Filella I, Gamon J (1995) Assessment of photosynthetic radiation use efficiency with spectral reflectance. New Phytol. https://doi.org/10.1111/j.1469-8137.1995.tb03064.x

Polívka T, Frank HA (2016) Spectroscopic investigation of carotenoids involved in non-photochemical fluorescence quenching. In: Demmig-Adams B, Garab G, Adams WW (eds) Non-Photochemical Quenching and Energy Dissipation in Plants, Algae and Cyanobacteria. Springer, New York, pp 203-228. https:// doi.org/10.1007/978-94-017-9032-1

Polívka T, Sundström V (2004) Ultrafast dynamics of carotenoid excited states-from solution to natural and artificial systems. Chem Rev 104:2021-2071. https://doi.org/10.1021/cr020674n

Ripullone F, Rivelli AR, Baraldi R et al (2011) Effectiveness of the photochemical reflectance index to track photosynthetic activity over a range of forest tree species and plant water statuses. Funct Plant Biol 38:177-186. https://doi.org/10.1071/FP10078

Romero E, Mozzo M, Van Stokkum IHM et al (2009) The origin of the low-energy form of photosystem I light-harvesting complex Lhca4: mixing of the lowest exciton with a chargetransfer state. Biophys J 96:L35-L37. https://doi.org/10.1016/j. bpj.2008.11.043

Ruban AV, Horton P (1995) An investigation of the sustained component of nonphotochemkal quenching of chlorophyll fluorescence 
in isolated chloroplasts and leaves of spinach. Plant Physiol 108:721-726. https://doi.org/10.1104/pp.108.2.721

Ruban AV, Young J, Horton P (1993) Induction of nonphotochemical energy dissipation and absorbance changes in leaves (evidence for changes in the state of the light-harvesting system of photosystem ii in vivo). Plant Physiol 102:741-750. https://doi.org/10.1104/ pp.102.3.741

Ruban AV, Berera R, Ilioaia C et al (2007) Identification of a mechanism of photoprotective energy dissipation in higher plants. Nature 450:575-579. https://doi.org/10.1038/nature06262

Ruban AV, Johnson MP, Duffy CDP (2012) The photoprotective molecular switch in the photosystem II antenna. Biochim Biophys Acta Bioenerg 1817:167-181. https://doi.org/10.1016/j. bbabio.2011.04.007

Scholes GD, Fleming GR (2006) Energy transfer and photosynthetic light harvesting. In: Berry RS, Jortner J (eds) Adventures in chemical physics: a special volume in advances in chemical physics. Wiley, New Jersey, pp 57-129

Scholes GD, Fleming GR, Olaya-Castro A, van Grondelle R (2011) Lessons from nature about solar light harvesting. Nat Chem 3:763-774. https://doi.org/10.1038/nchem.1145

van Grondelle R, Novoderezhkin VI (2006) Energy transfer in photosynthesis: experimental insights and quantitative models. Phys Chem Chem Phys 8:793-807. https://doi.org/10.1039/b514032c

Van Wittenberghe S, Alonso L, Verrelst J et al (2013) Upward and downward solar-induced chlorophyll fluorescence yield indices of four tree species as indicators of traffic pollution in Valencia. Environ Pollut 173:29-37. https://doi.org/10.1016/j.envpo 1.2012.10.003

Van Wittenberghe S, Alonso L, Verrelst J et al (2015) Bidirectional sun-induced chlorophyll fluorescence emission is influenced by leaf structure and light scattering properties-A bottomup approach. Remote Sens Environ 158:169-179. https://doi. org/10.1016/j.rse.2014.11.012

Van Wittenberghe S, Alonso L, Malenovský Z, Moreno J (2018) Photoprotection dynamics observed at leaf level from fast temporal reflectance changes. In: IEEE international symposium in geoscience and remote sensing (IGARSS), pp 5987-5990. https://doi. org/10.1109/igarss.2018.8518539

Vilfan N, Van der Tol C, Yang P et al (2018) Extending Fluspect to simulate xanthophyll driven leaf reflectance dynamics. Remote Sens Environ 211:345-356. https://doi.org/10.1016/j.rse.2018.04.012

Wientjes E, Roest G, Croce R (2012) From red to blue to far-red in Lhca4: how does the protein modulate the spectral properties of the pigments? Biochim Biophys Acta Bioenerg 1817:711-717. https://doi.org/10.1016/j.bbabio.2012.02.030

Xu D, Yue C, Gen-yun C (2015) Light-harvesting regulation from leaf to molecule with the emphasis on rapid changes in antenna size. Photosynth Res 124:137-158. https://doi.org/10.1007/s1112 0-015-0115-Z

Yamamoto Y, Aminaka R, Yoshioka M et al (2008) Quality control of photosystem II: impact of light and heat stresses. Photosynth Res 98:589-608. https://doi.org/10.1007/s11120-008-9372-4

Young AJ, Frank HA (1996) Energy transfer reactions involving carotenoids: quenching of chlorophyll fluorescence. J Photochem Photobiol B Biol 36:3-15

Publisher's Note Springer Nature remains neutral with regard to jurisdictional claims in published maps and institutional affiliations.

\section{Affiliations}

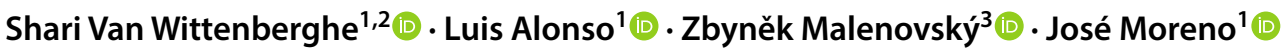

Luis Alonso

Luis.Alonso@uv.es

Zbyněk Malenovský

zbynek.malenovsky@gmail.com

José Moreno

Jose.Moreno@uv.es

1 Laboratory of Earth Observation, Image Processing Laboratory, University of Valencia, C/Catedrático José Beltrán, 2, 46980 Paterna, Valencia, Spain
2 Optics of Photosynthesis Laboratory, Institute

for Atmospheric and Earth System Research/Forest Sciences, Faculty of Agriculture and Forestry, University of Helsinki, 00014 Helsinki, Finland

3 Geography and Spatial Sciences, School of Technology, Environments and Design, University of Tasmania, Private Bag 76, Hobart, TAS 7001, Australia 